\title{
Kinetic Magnetic-Field Effect Involving the Small Biologically Relevant Inorganic Radicals $\mathrm{NO}$ and $\mathrm{O}_{2}{ }^{--}$
}

\author{
Tatiana Y. Karogodina, ${ }^{[a]}$ Igor G. Dranov ${ }_{\imath}^{[a]}$ Svetlana V. Sergeeva, ${ }^{[b]}$ Dmitry V. Stass, ${ }^{*[a]}$ and \\ Ulrich E. Steiner*[c]
}

Oxidation of dihydrorhodamine 123 (DHR) to rhodamine 123 $(\mathrm{RH})$ by oxoperoxonitrite ( $\left.\mathrm{ONOO}^{-}\right)$, formed through recombination of $\mathrm{NO}$ and $\mathrm{O}_{2}^{--}$radicals resulting from thermal decomposition of 3-morpholinosydnonimine (SIN-1) in buffered aerated aqueous solution at $\mathrm{pH} 7.6$, represents a kinetic model system of the reactivity of $\mathrm{NO}$ and $\mathrm{O}_{2}{ }^{--}$in biochemical systems. A magnetic-field effect (MFE) on the yield of RH detected in this system is explored in the full range of fields between 0 and $18 \mathrm{~T}$. It is found to increase in a nearly linear fashion up to a value of $5.5 \pm 1.6 \%$ at $18 \mathrm{~T}$ and $23^{\circ} \mathrm{C}\left(3.1 \pm 0.7 \%\right.$ at $\left.40^{\circ} \mathrm{C}\right)$. A theoretical framework to analyze the MFE in terms of the magnetic-field-enhanced recombination rate constant $k_{\text {rec }}$ of NO and $\mathrm{O}_{2}{ }^{-}$due to magnetic mixing of $\mathrm{T}_{0}$ and $\mathrm{S}$ spin states of the radical pair by the $\Delta g$ mechanism is developed, including estimation of magnetic properties ( $g$ tensor and spin relaxation times) of $\mathrm{NO}$ and $\mathrm{O}_{2}{ }^{--}$in aqueous solution, and calculation of the MFE on $k_{\text {rec }}$ using the theoretical formalism of Gorelik at al. The factor with which the MFE on $k_{\text {rec }}$ is translated to the MFE on the yield of $\mathrm{ONOO}^{-}$and $\mathrm{RH}$ is derived for various kinetic scenarios representing possible sink channels for $\mathrm{NO}$ and $\mathrm{O}_{2}{ }^{\circ}$. With reasonable assumptions for the values of some unknown kinetic parameters, the theoretical predictions account well for the observed MFE.

\section{Introduction}

Many biological species, including mammals, birds, reptiles, amphibians, fish, crustaceans, and insects, are sensitive to magnetic fields and can even use the geomagnetic field for navigation. ${ }^{[1-5]}$ The biophysical, biochemical, and neuronal mechanisms of magnetic-field perception are still mostly hypothetical. Usually, one of the three following basic principles is favored: biogenic magnetite particles, electromagnetic induction, or spin chemistry. The last-named not only provides possible mechanisms for biomagnetic sensing, but is also of interest as a diagnostic tool for assigning biochemical reaction pathways.

The field of spin chemistry ${ }^{[6-9]}$ has been developed during the last 40 years to the level of a mature discipline with a wide scope of experimental methods and a solid coherent theoretical basis. It has also found interesting applications in various biochemical processes in vitro and in vivo. A central paradigm of spin chemistry is the radical-pair mechanism. If radical pairs appear as intermediates of chemical reactions, the alignment of the unpaired spins-whether they are antiparallel (singlet) or parallel (triplet)-determines the rates of recombination into reaction channels of different multiplicity or dissociation of the radical pair. Therefore, any interaction that affects the electron spins, including an external magnetic field, can modify the reaction rates and/or yields into different reaction channels.

Radical pairs are involved in many biochemical reactions. ${ }^{[10]}$ The primary radical pair in photosynthesis has figured most prominently in the development of spin chemistry and its applications to biological systems. ${ }^{[7,11,12]}$ Radical pairs are often involved in enzymatic oxidation or reduction reactions. Magnet-

ic-field effects (MFEs) have been detected in the photolysis of adenosylcobalamine ${ }^{[13]}$ and in the horseradish peroxidase catalyzed oxidation of $\mathrm{NADH}^{\left[{ }^{[14]}\right.}$ In the latter case, the magnetically sensitive intermediate is assumed to be a pair of a spin-triplet species (high-spin $\mathrm{Fe}^{2+}$ complex in the reduced ferroperoxidase) and a spin-doublet species (NAD* radical). The radicalpair mechanism is also responsible for kinetic effects that can be found when substituting nonmagnetic by magnetic nuclear isotopes (magnetic isotope effect) ${ }^{[7,15]}$ Thus, the occurrence of such effects is diagnostic of the involvement of radical pairs in a chemical mechanism. Surprisingly, magnetic isotope effects were recently reported for ATP synthesis by phosphorylating enzymes, ${ }^{[14]}$ where the involvement of radical pairs would be counterintuitive according to traditional biochemical concepts.

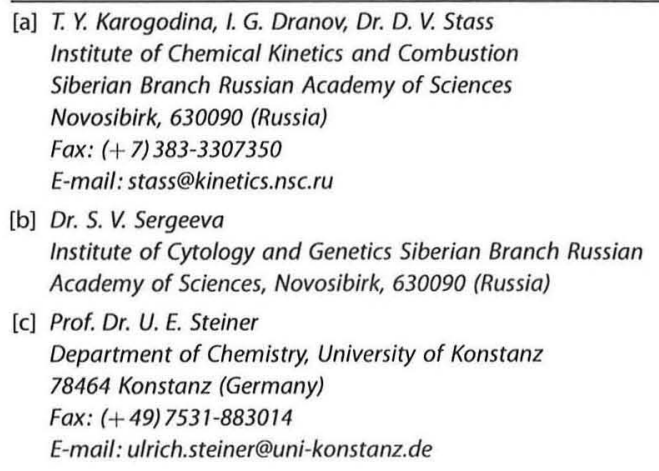


A number of small inorganic radicals have proven to be of high significance in medical biochemistry. Thus, inflammatory processes are intimately related to the oxygen radicals superoxide anion $\mathrm{O}_{2}^{--}$, the hydroperoxide radical $\mathrm{HO}_{2}{ }_{2}$, and the hydroxyl radical ${ }^{\circ} \mathrm{OH} .{ }^{[16]}$ The role of nitric oxide $\mathrm{NO}$ as a messenger molecule ${ }^{[17-18]}$ important in immune defense, cell communication, and vasodilatory action has become prominent during the last 30 years and has attracted broad public interest since the Nobel prize in medicine in 1998 was awarded for discoveries in this field. In vivo recombination of biogenic $\mathrm{O}_{2}{ }^{-}$ and NO to form oxoperoxonitrate $\left(\mathrm{ONOO}^{-}\right)^{[19]}$ has been recognized as an important route to the oxidation of many biochemical targets. ${ }^{[20-22]}$ Since a radical-pair recombination is involved, it would be interesting to find out whether such oxidation reactions with $\mathrm{ONOO}^{-}$are sensitive to a magnetic field. The focus of interest would not be, in the first place, to account for external MFEs on biological systems, but rather to apply such MFEs as diagnostic tools for identifying the mechanism leading to some biochemical oxidation products.

What kind of MFE should one expect for the recombination of free radicals if they recombine to form a diamagnetic product? Recombination requires that the radical pair is in a singlet spin state. For freely recombining radicals, this will be the case for one-fourth of all encounters, while for three-fourths of the encounters the overall electron spin will correspond to a triplet state and therefore will be unreactive in the first place. However, during the overall time period of an encounter, which may involve several small separations and recollisions on a short timescale, there is a finite probability of a multiplicity change due to locally different interactions of the two radical spins with internal or external magnetic fields.

As an internal magnetic field, the ${ }^{14} \mathrm{~N}$ hyperfine interaction (ca. $22 \mathrm{MHz}^{[23]}$ ) must be considered too weak to be effective during the very short encounter lifetime of the NO radical with the $\mathrm{O}_{2}{ }^{--}$radical in a low-viscosity environment. However, spinorbit coupling in small linear radicals causes strong alignment of the spin with the molecular axis. Thereby, the spin will be reoriented at a rate comparable to molecular rotation times. Although, in solution, the electrical interaction with the solvent shell will partially quench the orbital angular momentum, and thereby decrease the spin-orbit coupling effect, it can be anticipated that spin relaxation and molecular reorientation will occur on a similar timescale.

The electron spin relaxation time of a radical represents its spin memory time. If it is comparable to the timescale of an encounter, radical pairs that initially come together with triplet spin alignment will also have a chance of reacting during the encounter. An external MFE on the overall recombination rate can ensue only if the external magnetic field enhances the triplet-singlet conversion process in the radical pair to such an extent that it becomes comparable to or faster than the spin relaxation process. ${ }^{[24]}$ In spin chemistry, it is well known that external magnetic fields can induce fast triplet-singlet interconversion in a radical pair by the so-called $\Delta g$ mechanism if the two radicals differ in their $g$ factors and the external magnetic field is strong. ${ }^{[7]}$ In such cases, kinetic MFEs ensue even if spin relaxation takes place during a few picoseconds. ${ }^{[25]} \mathrm{Al}-$ though the $g$ factors of $\mathrm{NO}$ and $\mathrm{O}_{2}{ }^{--}$in liquid solution are not known, because these radicals escape EPR detection due to their fast spin relaxation, the difference in their $g$ factors may be on the order of unity, so that in fields of several Tesla triplet-singlet mixing may become fast enough to affect the recombination rate constant.

However, so far spin-chemical effects with such small radicals have mostly escaped detection. In two theoretical papers, though, it was controversially discussed whether the $\mathrm{O}_{2}^{--}$radical may be involved in the hypothetical radical-pair mechanism on which the geomagnetic compass of some migratory birds is suggested to be based. ${ }^{[26-27]}$ A spin-chemical effect of the $\cdot \mathrm{OH}$ radical has been invoked to interpret chemically induced dynamic electron polarization (CIDEP) effects in some systems. ${ }^{[28-29]}$ To the best of our knowledge, the only statistically reliable MFE on a product yield was reported in a single field of $4.7 \mathrm{~T}$ for an in vitro model system mimicking biological in situ generation of $\mathrm{ONOO}^{-}$by recombination of $\mathrm{NO}$ and $\mathrm{O}_{2}{ }^{-}$ and irreversible oxidation of a substrate by $\mathrm{ONOO}^{-} .{ }^{130]}$ The overall reaction system starting with the formation of $\mathrm{NO}$ and $\mathrm{O}_{2}^{--}$by thermal decomposition of 3-(4-morpholino)sydnonimine (SIN-1) in aerated solution is rather complex. In this work, we characterize in more detail the kinetic sequence leading to the final oxidation product rhodamine $123(\mathrm{RH})$ formed by oxidation of of dihydrorhodamine 123 (DHR) via the intermediate $\mathrm{ONOO}^{-}$, and explore the magnetic-field dependence of the effect in a field range up to $18 \mathrm{~T}$. Based on the experimental findings we develop a theoretical framework accounting for the MFE in terms of the spin chemistry of the $\mathrm{NO} / \mathrm{O}_{2}{ }^{-}$radical pair.

\section{Results}

\subsection{Reaction System}

In the literature, interest in the decay kinetics of SIN-1 originated from pharmaceutical interest in the metabolites of molsidomin (the $\mathrm{N}$-ethoxycarbonyl derivative of $\mathrm{SIN}-1$ ), a vasodilatory drug that has been in use since 1977. A first kinetic study was presented in 1979 by Asahi et al. ${ }^{[3]}$ In this study, the basic steps of SIN-1 transformation were identified in analogy to earlier investigations on sydnonimines (Scheme 1). ${ }^{[32-33]} \mathrm{SIN}-1$ is easily protonated $\left(\mathrm{p} K_{\mathrm{a}}=9.1\right.$ at $\left.25^{\circ} \mathrm{C}\right) .^{[31]}$ Thus, at $\mathrm{pH} 7-8$ the free base is only present as a minor fraction. It undergoes ring opening to 2-[(4-morpholino)nitrosoamino]acetonitrile (SIN-1A) with a rate constant $k^{\prime}$ of about $0.2 \mathrm{~min}^{-1}$ at $\mathrm{pH}<7.5$. The final product is 2-[(4-morpholinoimino]acetonitrile (SIN-1C). Elimination of NO was demonstrated later ${ }^{[34]}$ as well as the role of oxygen as a one-electron oxidant of SIN-1A producing its radical cation and the superoxide ion $\mathrm{O}_{2} \cdot{ }^{\cdot-[35-36]}$

The thermal decomposition of SIN-1 thus becomes a source of $\mathrm{NO}$ and $\mathrm{O}_{2}{ }^{--}$radicals. These can recombine in a nearly diffusion controlled reaction. Values of the recombination rate constant derived from pulse radiolysis experiments are in the region of $(3-6) \times 10^{9} \mathrm{M}^{-1} \mathrm{~s}^{-1},{ }^{[37]}$ whereas flash photolysis experiments on $\mathrm{ONOO}^{-}$yielded a recombination rate constant of $1.6 \times 10^{10} \mathrm{M}^{-1} \mathrm{~s}^{-1}$ [38] $^{38}$ 


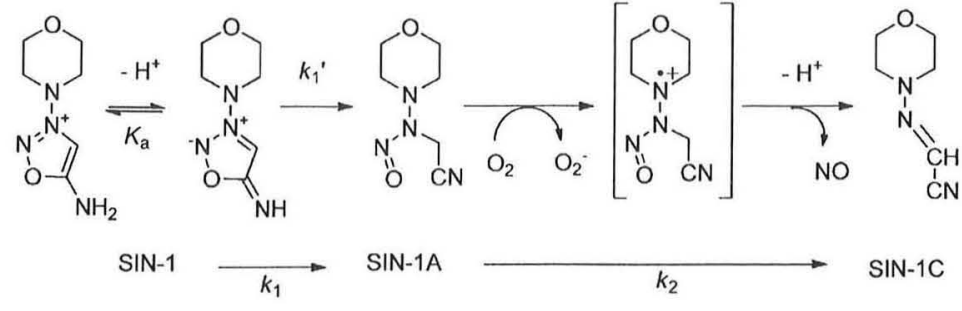

Scheme 1. Mechanism of thermal decomposition of SIN-1 in aerated solution.

Although recombination of $\mathrm{NO}$ and $\mathrm{O}_{2}^{-{ }^{-}}$is very fast, it is unlikely that $\mathrm{NO}$ and $\mathrm{O}_{2}{ }^{--}$radicals created in the decay of the same SIN-1 molecule will recombine with each other (geminate recombination). The reason for this is the delay time between formation of $\mathrm{O}_{2}{ }^{--}$and the SIN-1A radical cation and formation of free NO, which requires a prior deprotonation step at an $\mathrm{sp}^{3}$-hybridized carbon atom. The activation energy of this reaction step is probably quite high. Furthermore, there is no strong base to accept the proton. Hence it seems safe enough to assume that NO release takes longer than $1 \mathrm{~ns}$. During such a time span, the mean distance of separation of the $\mathrm{O}_{2}{ }^{--}$radical would amount to $\Delta r \approx \sqrt{6 D t}=27 \AA^{[39]}$ if a diffusion constant for $\mathrm{O}_{2}^{-{ }^{-}}$of $1.2 \times 10^{-5} \mathrm{~cm}^{2} \mathrm{~s}^{-1}$ is used. ${ }^{[40]}$ Assuming for NO and $\mathrm{O}_{2}{ }^{--}$a recombination radius of $3.6 \AA_{1}^{[40]}$ the probability that the two radicals would recombine with each other in a diffusion-controlled reaction would be $3.6 / 27=0.13,{ }^{[41]}$ an estimate which probably represents an upper limit.

To assess the kinetics of $\mathrm{ONOO}^{-}$formation under the conditions of our experiments, the thermal decay of SIN-1 was studied spectrophotometrically (Figure 1). The observed UV/Vis spectra represent the time-dependent superposition of the specific contributions of SIN-1 (protonated form), SIN-1A, and SIN-1C. By applying a global fit for six characteristic wavelengths (see Experimental Section and Supporting Information), the rate constants $k_{1}=0.008 \mathrm{~min}^{-1}$ and $k_{2}=0.018 \mathrm{~min}^{-1}$ (see Scheme 1) could be assigned. Figure 2 shows the time dependence of the concentrations of the three species derived from the global kinetic fit. Since $\mathrm{NO}$ and $\mathrm{O}_{2}{ }^{-}$appear synchro-

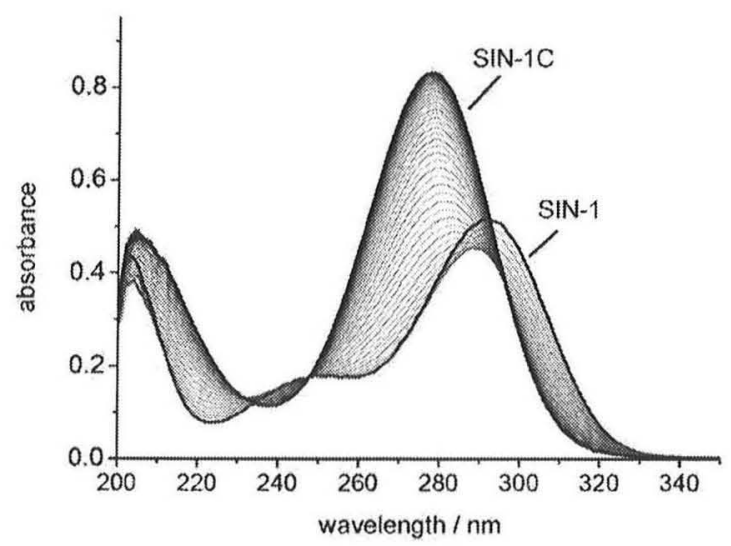

Figure 1. Series of absorption spectra for a SIN-1 solution $(50 \mu \mathrm{M})$ at $\mathrm{pH} 7.6$. The spectra were taken at time intervals of $10 \mathrm{~min}$. nously with SIN-1C and the recombination reaction of these radicals is fast, formation of $\mathrm{ONOO}^{-}$is expected to match formation of SIN-1C.

The formation of $\mathrm{ONOO}^{-}$has been conveniently measured by means of its facile reaction with reduced fluorophores such as dihydrorhodamine 123 (DHR) or dihydrofluorescein to form their oxidized forms, which can be easily detected by their strong fluorescence or absorption in the visible. ${ }^{[33,42]}$ In this work, we used DHR as a probe for $\mathrm{ONOO}^{-}$. In this regard it is important that DHR does not directly react with the precursor radicals

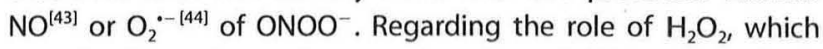
may be formed on disproportionation of $\mathrm{O}_{2}{ }^{--}$, it has been stated in the literature that "DHR does not react with $\mathrm{H}_{2} \mathrm{O}_{2}$ alone $^{\prime \prime[44]}$ (i.e., in the absence of catalysts or enzymes) or "in the absence of enzymes $\mathrm{H}_{2} \mathrm{O}_{2}$ oxidized DHR slowly". ${ }^{\text {[45] }}$ The effective second-order rate constant for disproportionation of $\mathrm{O}_{2}^{--}$at $\mathrm{pH} 7.6$ is $1.5 \times 10^{5} \mathrm{M}^{-1} \mathrm{~s}^{-1}$ [see Eqs. (34) and (35)]. This is much smaller than the value for the diffusion-controlled recombination of $\mathrm{NO}$ and $\mathrm{O}_{2}{ }^{--}{ }^{[40]}$ Hence, the yield of $\mathrm{H}_{2} \mathrm{O}_{2}$ in SIN1 decomposition will be low, and we consider the fraction of oxidation of $\mathrm{DHR}$ by $\mathrm{H}_{2} \mathrm{O}_{2}$ as negligible.

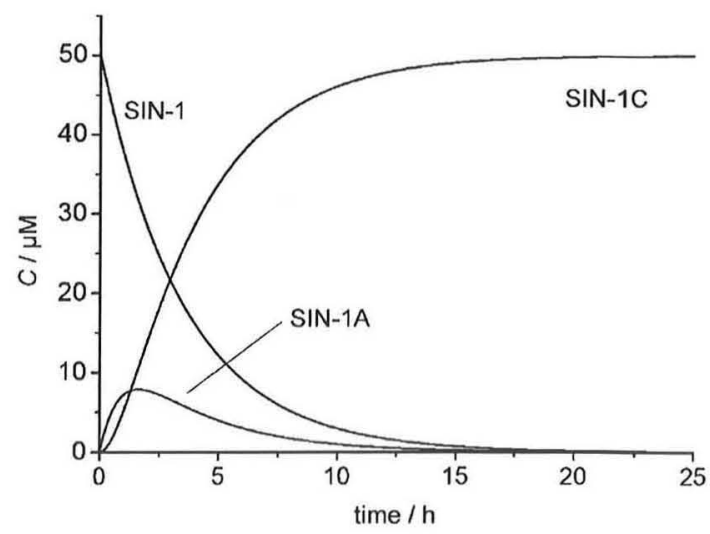

Figure 2. Time profiles of various SIN-1 species using the rate constants obtained by the global fit of the spectra in Figure 1.

In the literature, there is some controversy about the mechanism of the reaction between $\mathrm{DHR}$ and $\mathrm{ONOO}^{-}$. A direct reaction between these species is assumed by Kooy et al. ${ }^{[43]}$ and by Kumar et al. ${ }^{[46]}$ In the latter work, a second-order rate constant of $8.2 \times 10^{3} \mathrm{M}^{-1} \mathrm{~s}^{-1}$ was determined at $\mathrm{pH} 7$. On the other hand, from their kinetic observations other authors concluded that no direct reaction occurs between $\mathrm{DHR}$ and $\mathrm{ONOO}^{-}$. While Glebska et al. ${ }^{[47]}$ assumed the intermediacy of a protonated complex between these two reactants, Jourd'heuil et al. ${ }^{[48]}$ advocated the role of $\mathrm{NO}_{2}$ and $\mathrm{OH}$, the radical decay products of $\mathrm{ONOOH}$, as active oxidizing species. 


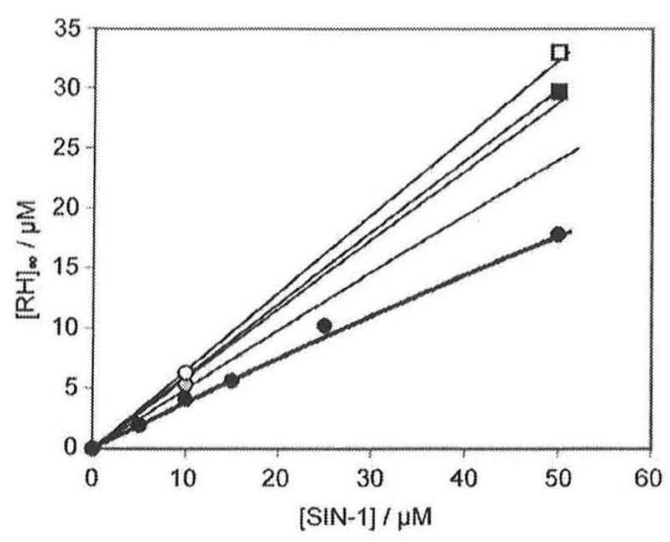

Figure 3. Yield of RH as a function of $\mathrm{SIN}-1$ concentration for various concentrations of DHR: data points $50 \mu \mathrm{M}(\bullet), 100 \mu \mathrm{M}(\bullet), 200 \mu \mathrm{M}(0), 250 \mu \mathrm{M}$ (घ), $500 \mu \mathrm{M}(\square)$. The solid lines $(\longrightarrow$ ) result from kinetic simulations with Equation (4). They correspond (from bottom to top) to the concentrations: $50 \mu \mathrm{M}$ (bold line), $100 \mu \mathrm{M}, 200 \mu \mathrm{M}, 250 \mu \mathrm{M}, 500 \mu \mathrm{M}$ (for details, see text).

To obtain a suitable kinetic representation of RH formation under our experimental conditions, we measured the yield of $\mathrm{RH}$ for various concentrations of SIN-1 and DHR. To detect the total yield, the $\mathrm{RH}$ signal was measured after a reaction time of $24 \mathrm{~h}$. The results are shown in Figure 3. They can be kinetically simulated on the basis of the following reaction sequence [Eqs. (1)-(3)]:

$\mathrm{SIN}-1 \stackrel{F(t)}{\longrightarrow} a_{1} \mathrm{ONOO}^{-}$

$\mathrm{ONOO}^{-} \stackrel{k_{3}}{\longrightarrow}$ decomposition

$\mathrm{ONOO}^{-}+\mathrm{DHR}^{k_{4}} \underset{a_{2}}{\mathrm{RH}}+\left(1-a_{2}\right) \mathrm{DHR}$

In reaction (1), the factor $a_{1}$ accounts for the fact that $\mathrm{ONOO}^{-}$is not necessarily formed with $100 \%$ efficiency from $\mathrm{SIN}-1$ because of other decay channels of $\mathrm{NO}$ and $\mathrm{O}_{2}{ }^{--}$. As shown in the Supporting Information, the final value of the yield $[\mathrm{RH}]_{\infty}$ does not depend on the rate $F(t)$ of reaction (1), and hence the time dependence of this reaction step need not be explicitly taken into account.

The decomposition of $\mathrm{ONOO}^{-}$must be assumed to involve the protonated form and its dissociation into the radicals $\mathrm{NO}_{2}$ and $\mathrm{OH}$. It is assigned a reaction constant $k_{3}$ of about $0.32 \mathrm{~s}^{-1}$ at $\mathrm{pH} 7.6 .{ }^{[47]}$ The reaction between $\mathrm{ONOO}^{-}$and DHR is assigned an effective second-order rate constant $k_{4}$. Formation of $\mathrm{RH}$ occurs mainly through disproportionation of the radical $\mathrm{DHR}^{\circ+}$, which is assigned a yield factor $a_{2}$. From this sequence of reactions, one can derive the implicit Equation (4) for the yield $[\mathrm{RH}]_{\infty}$ (for the derivation of Equation (4), see the Supporting Information):

$[\mathrm{RH}]_{\infty}=a_{1} a_{2}[\mathrm{SIN}-1]_{0}+\frac{k_{3}}{k_{4}} \ln \left(1-\frac{[\mathrm{RH}]_{\infty}}{[\mathrm{DHR}]_{0}}\right)$

A global fit of the data points in Figure 3 yields $a_{1} a_{2}=0.7$ and $k_{3} / k_{4}=40 \mu \mathrm{m}$. Assuming for $k_{3}$ a value of $0.32 \mathrm{~s}^{-1}$ leads to $k_{4}=0.81 \times 10^{4} \mathrm{M}^{-1} \mathrm{~s}^{-1}$, in fair agreement with the value given in ref. [46].

\subsection{Magnetic-Field Effect}

When the reaction of the SIN-1/DHR system takes place in a magnetic field, a field dependence of the yield of RH is observed. In each experiment, the magnet was loaded with five or six equivalent samples in combination with an equivalent set of five or six control samples outside the magnet (for details, see Experimental Section) Typical results of such an experiment are shown in Figure 4. The RH absorbance values from the samples in the field are higher than from the control samples outside the field.

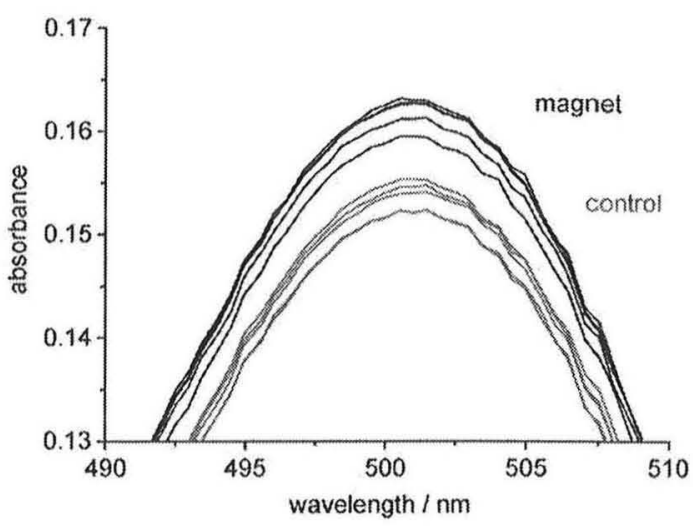

Figure 4. Absorbance curves for a typical run of a magnetic-field experiment. $[\mathrm{SIN}-1]=50 \mu \mathrm{M},[\mathrm{DHR}]=50 \mu \mathrm{M}, \mathrm{pH} 7.6$. In this case the average MFE (for definition, see text) is $5.3 \%$.

To analyze the size of the effect and the accuracy of its measurement quantitatively, we define the MFE $\varphi_{Y}$ on the yield of $\mathrm{RH}$ as Equation (5):

$\varphi_{\mathrm{Y}} \equiv \frac{\bar{A}_{\mathrm{m}}-\bar{A}_{\mathrm{c}}}{\bar{A}_{\mathrm{c}}}$

where $\bar{A}_{\mathrm{m}}$ is the average value of the maximum absorbance in the field and $\bar{A}_{\mathrm{c}}$ the corresponding value of the control.

To reduce the statistical error margin, several such runs with five or six samples in each were performed for each field value. The statistics of these experiments are shown for two field values in Figure 5.

The magnetic-field dependence of the field effect is plotted in Figure 6. Each data point represents the average of $m$ experiments, each with $n$ samples in the sample holder in the magnet and in the control. The error bars represent standard deviations of the averages of the $m$ experiments (see Experimental Section). The solid lines represent theoretical fits, as described in the Discussion. 


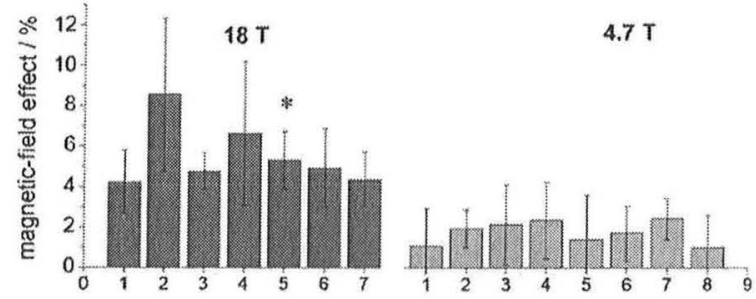

Figure 5. Histograms of several sets of MFE experiments at 18 and $4.7 \mathrm{~T}$. The columns indicate the average field effect in each experiment and the error bars the standard deviation. The column marked by the asterisk represents the measurement shown in Figure 4.

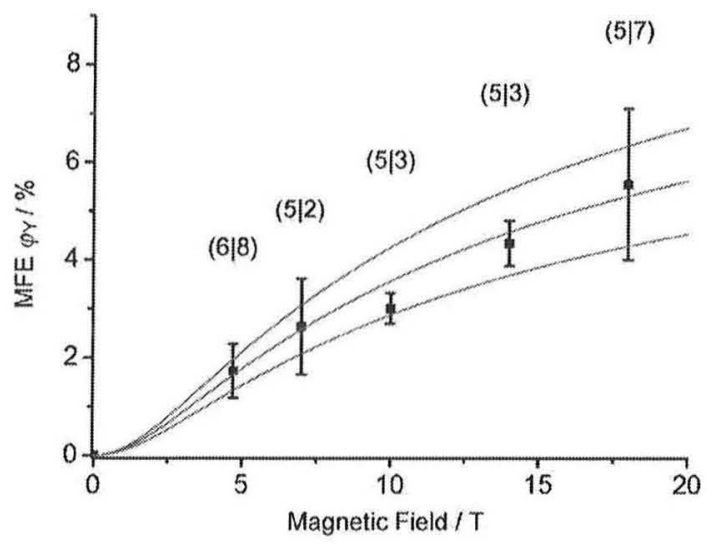

Figure 6. MFE on the yield of RH in solutions with $[\mathrm{SIN}-1]=50 \mu \mathrm{M}$, $[D H R]=50 \mu \mathrm{M}, \mathrm{pH} 7.6$ as a function of magnetic field. The pair of numbers $(n \mid m)$ at each data point represents the number $m$ of experiments each with a number $n$ of samples. The lines represent theoretical simulations calculated by assuming the following standard values of the reaction parameters: $R=3.65 \AA, \delta=0.3 \AA, \tau_{\mathrm{c}}=1.4 \mathrm{ps}, x=1$, and $k_{\mathrm{r}}=2 \times 10^{11} \mathrm{~s}^{-1}$ and $q$ values ranging from 0.25 (lowest curve), through 0.31 (middle curve), to 0.37 (upper curve); for details, see Discussion.

\subsection{Temperature Dependence}

While the main body of data was determined at a temperature of $23^{\circ} \mathrm{C}$, several experiments were carried out at an elevated temperature of $40^{\circ} \mathrm{C}$ at the highest field of $18 \mathrm{~T}$. The resulting MFE amounted to $3.1 \pm 0.7 \%$, as compared to $5.5 \pm 1.6 \%$ at $23^{\circ} \mathrm{C}$.

\section{Discussion}

\subsection{Characterization of the MFE}

The main characteristics of the observed MFE are 1) it requires very high magnetic fields, 2 ) it rises monotonically, showing no saturation up to $18 \mathrm{~T}, 3$ ) it enhances a product yield. These features are characteristic of the $\Delta g$ mechanism in radical pairs undergoing unrestricted diffusion. ${ }^{[7]}$ In this mechanism, the different $g$ factors of the two radicals cause different Larmor precessions of the electronic spins about the direction of the external magnetic field, which thereby lead to a conversion between the $T_{0}$ and $S$ spin states of the radical pair at a rate/frequency proportional to the external magnetic field ${ }^{[49]}[\mathrm{Eq}$. (6)]:
$\Delta \omega / \mathrm{s}^{-1} \mathrm{~T}^{-1}=8.79 \times 10^{10} \Delta g B_{0}$

which corresponds to an effective Larmor dephasing time $\tau_{\mathrm{L}}$ of [Eq. (7)]:

$\tau_{\mathrm{L}} / \mathrm{ps} \cdot \mathrm{T}=11.4 / \Delta g B_{0}$

The positive MFE on the yield of a diamagnetic (i.e., singlet) product indicates that the radical-pair precursor is predominantly of triplet multiplicity, because in that case accelerating the $T_{0} \leftrightarrow S$ process by a magnetic field will support formation of the recombination product. The requirement of very high magnetic fields could indicate that either $\Delta g$ is rather small, ${ }^{[0]}$ or that at least one of the two radical species is subject to a fast, magnetic-field-independent electron spin relaxation process because, in order to appear as a significant kinetic effect, the magnetic-field-dependent contribution to the rate of the $T \leftrightarrow S$ conversion process should become comparable to the typical rate constant of spin relaxation in the radical-pair system. ${ }^{[25]} \mathrm{As}-$ suming, for example, a $\Delta g$ value on the order of unity, a magnetic field of $10 \mathrm{~T}$ corresponds to a Larmor dephasing time as short as about $1 \mathrm{ps}$.

In view of the crucial role of the radicals $\mathrm{NO}$ and $\mathrm{O}_{2}{ }^{-{ }^{-}}$in the overall reaction mechanism, it is suggested to scrutinize the spin chemical option of the $\mathrm{NO} / \mathrm{O}_{2}^{-}$radical pair to account for the observed MFE. However, due to the not yet fully understood complexity of the overall reaction mechanism on the way from SIN-1 to RH, the hypothetical character of this approach must be emphasized. Nevertheless, the characteristics of the MFE specified above seem to indicate that at least one of these small inorganic radicals is involved in one or more types of radical pairs that may be responsible for the observed effects.

\subsection{Magnetic Properties of $\mathrm{NO}$ and $\mathrm{O}_{2}{ }^{\circ}$}

The $\mathrm{NO}$ and $\mathrm{O}_{2}^{-}$radicals both have an incompletely filled molecular $2 \pi^{*}$ shell. In NO, it is populated by just one electron, while in $\mathrm{O}_{2}^{--}$there are three electrons or, equivalently, one hole. For isolated, nonrotating molecules, the electronic ground term is split due to spin-orbit coupling with a ${ }^{2} \Pi_{1 / 2}$ and ${ }^{2} \Pi_{3 / 2}$ state as the lowest for $\mathrm{NO}$ and $\mathrm{O}_{2}{ }^{-}$, respectively. In these states, the electron (hole) spin is strictly coupled to the molecular axis, as follows from the $g$-tensor values of $g_{\|}=0$, $g_{\perp}=0$ for $\mathrm{NO}$ and $g_{\|}=4, g_{\perp}=0$ for $\mathrm{O}_{2}{ }^{--}$In solution, the rotational symmetry of the orbitals around the molecular axis ( $z$ direction) is broken by the electrostatic interaction with the environment. Thereby, the orbital angular momentum is more or less quenched. For a finite splitting $\Delta$ between the $2 \pi_{x}^{*}$ and the $2 \pi_{y}^{*}$ orbitals, the resulting energy difference between ground and first excited state of the radicals is given by $\sqrt{\lambda^{2}+\Delta^{2}}$ (see Figure 7).

If spin-orbit coupling with terms of $\sigma$ symmetry is neglected, the $g$ tensors for the ground state are given by Equations (8) and (9): ${ }^{[51]}$ 


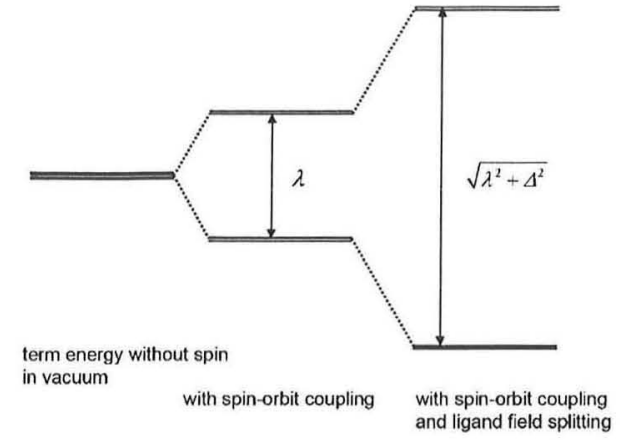

Figure 7. Spin-orbit coupling and ligand-field effects on level splitting and energies of the lowest two Kramers doublets in linear radicals.

$$
\begin{aligned}
& g_{z z}=g_{\mathrm{e}}-2 \frac{\lambda}{\sqrt{\lambda^{2}+\Delta^{2}}} \\
& g_{x x}=g_{y y}=g_{e} \frac{\Delta}{\sqrt{\lambda^{2}+\Delta^{2}}}
\end{aligned}
$$

where $g_{\mathrm{e}}$ is the $g$ factor of the free electron. In Equation (8), the sign of $\lambda$ must be taken into account. It is positive $\left(120 \mathrm{~cm}^{-1}\right)$ for NO, and negative $\left(-160 \mathrm{~cm}^{-1}\right)$ for $\mathrm{O}_{2}{ }^{-} \cdot{ }^{-[52]}$

The EPR spectra of the two radicals have only been observed in solid media and at cryogenic temperatures. ${ }^{[53]}$ For NO, some studies with zeolites as host material were reported. ${ }^{[54-55]}$ Based on a spin-orbit coupling constant of $120 \mathrm{~cm}^{-1}$, values of 1.77-1.86 for $g_{\|}$and 1.97-1.99 for $g_{\perp}$ in a study conducted at $77 \mathrm{~K}$ by Gardner and Weinberger ${ }^{[54]}$ have been attributed to $\Delta$ values of $1000-1600 \mathrm{~cm}^{-1}$. In a more recent study using X-, Q-, and W-band EPR at $10 \mathrm{~K}$, Rudolf et al. ${ }^{[55]}$ found $g_{\|}$ $=1.85$ and 1.88 and $g_{\perp} \approx 1.99$, leading to $\Delta$ values of 1300 and $2100 \mathrm{~cm}^{-1}$, respectively. For $\mathrm{O}_{2}{ }^{--}$early studies in alkali metal halide host crystals were reported by Känzig and Zeller. ${ }^{[51,56]}$ Depending on the particular type of host, $g_{\|}$values ranging between 2.2 and 2.5 and $g_{\perp}$ values between 1.93 and 1.97 were observed. By using $\lambda=-160 \mathrm{~cm}^{-1}$, corresponding values for $\Delta$ between about 400 and $900 \mathrm{~cm}^{-1}$ can be deduced. EPR of $\mathrm{O}_{2}{ }^{--}$has been also reported in $\mathrm{EtOH}$ glass at $95 \mathrm{~K}^{[57]}$ and in $\mathrm{D}_{2} \mathrm{O}$ glass at $4.2 \mathrm{~K}$ with $g_{\|} \approx 2.07$ and $g_{\perp}$ close to $g_{\mathrm{e}^{.}}{ }^{[58]}$ These values indicate rather high $\Delta$ values of $>4000 \mathrm{~cm}^{-1}$. In protic solvents $\mathrm{H}$-bonding to $\mathrm{O}_{2}^{--}$is probably of major importance. Evidence of cluster formation of $\mathrm{O}_{2}{ }^{--}$with four $\mathrm{H}_{2} \mathrm{O}$ molecules was reported from a study employing IR cluster spectroscopy and ab initio calculations. ${ }^{\left[{ }^{[9]}\right.}$

From these observations we conclude that in liquid aqueous solution, too, the $g$ factors of the two radicals will significantly deviate from the value $g_{\mathrm{e}}$ of a free electron. However, due to the much stronger, hydrogen bond type interaction of the solvent with $\mathrm{O}_{2}^{--}$, this deviation will be more moderate for that radical than for NO. In the latter, the ligand-field splitting in liquid solution at room temperature may be significantly smaller than observed in low-temperature solid matrices, because the fluctuation of solvation is much faster and anisotropic interactions may be largely averaged out. Hence, assuming a $\Delta g$ value between the two radicals on the order of unity does not seem unrealistic. In that case, according to Equation (7), we may expect a Larmor dephasing time ( $\mathrm{T}_{0} / \mathrm{S}$ mixing time) $\tau_{\mathrm{L}}$ of about $1 \mathrm{ps}$ at a field of $10 \mathrm{~T}$. This is short enough to induce appreciable kinetic effects during a radical-pair cage time of typically several tens of picoseconds. With radical pairs involving "normal" organic radicals, for which $\Delta g$ typically is on the order of less than 0.01, such effects could never be achieved. However, whether the magnetic-field-dependent $T_{0} / S$ mixing becomes kinetically relevant depends not only on $\tau_{\mathrm{L}}$ but also on the spin memory time of the radical pair, as determined by the time constants of spin relaxation.

\subsection{Spin Relaxation}

In liquid solution, no EPR spectrum of either $\mathrm{NO}$ or $\mathrm{O}_{2}^{--}$has been observed. This fact indicates that spin relaxation in these radicals becomes too fast as they are allowed to rotate. The most obvious explanation for this must be sought in spin relaxation mechanisms related to $g$-tensor anisotropy. Individual spin relaxation in each of the two radicals of a radical pair also affects the overall spin state of the pair. Longitudinal relaxation (spin-lattice relaxation, characteristic time $T_{1}$ ) leads to $T_{ \pm}$ $\rightleftarrows\left(S, T_{0}\right)$ relaxation and transversal relaxation (phase relaxation, characteristic time $T_{2}$ ) leads to $S \rightleftarrows T_{0}$ relaxation. The latter process adds to the $\Delta g B_{0}$-induced $\mathrm{S} \leftrightarrow \mathrm{T}_{0}$ coherent spin-mixing process.

There are two ways by which tumbling of the radicals will stochastically modulate the spin Hamiltonian and thereby cause electron spin relaxation: spin-rotational interaction (sri) and $g$-tensor anisotropy (gta). For spin-rotational relaxation one has Equation (10): $:^{[60]}$

$\frac{1}{\tau_{\mathrm{s}}}=\frac{1}{T_{1}}=\frac{1}{T_{2}}=\overline{\frac{\delta g^{2}}{9}} \frac{1}{\tau_{\mathrm{c}}}$

where $\overline{\delta g^{2}}$ is given by Equation (11):

$\overline{\delta g^{2}}=\left(g_{\|}-g_{\mathrm{e}}\right)^{2}+2\left(g_{\perp}-g_{\mathrm{e}}\right)^{2}$

and $g_{\mathrm{e}}$ is the $g$ factor of the free electron.

The orientational correlation time $\tau_{\mathrm{c}}$ applying to secondorder tensorial quantities can be calculated by Equation (12): ${ }^{[61]}$

$\tau_{\mathrm{c}}=\frac{4 \pi R^{3} \eta}{3 k_{\mathrm{B}} T} f$

where $R$ is the van der Waals radius of the radical and $\eta$ the viscosity of the solvent. Without the factor $f$, this equation is valid for so-called "stick" conditions, where it is assumed that the solvent sticks to the surface of the rotating spherical particle. Its validity is restricted to particles that are considerably larger than the solvent molecules. In that case the factor $f$ is approximately unity. However, for small molecules, this factor is considerably less than expected from stick boundary conditions, and rather close to that expected from slip boundary 
conditions. ${ }^{[61]}$ Under slip boundary conditions deviation of the molecule from perfect spherical shape is essential. ${ }^{[62]}$

It is a characteristic property of the sri relaxation mechanism that $T_{1}=T_{2}$ and that these relaxation times are independent of an external magnetic field as long as the correlation time of molecular angular momentum is shorter than the Larmor period, a condition that will break down only at magnetic fields much higher than those applied herein.

Equation (10) is not valid for large values of $\overline{\delta g^{2}}$. It has been shown in references $[63,64]$ that the effect of molecular rotation captured in Equation (10) can be understood as a consequence of "adiabatic rotation of effective spin" (ARES), that is, the trajectory of effective spin orientations (the sequence of states adopted in the $2 \times 2$ spin space of the Kramers doublet) is a unique function of the sequence of positions adopted in the orientational space of the molecular axis, irrespective of the values of angular velocity at which the molecule traverses this trajectory. The rigidity at which the (effective) spin follows the molecular axis is defined by the $\gamma$ tensor, and $\overline{\delta g^{2}}$ in Equation (10) should be replaced by $\overline{\delta \gamma^{2}}$. For weak spin-orbit coupling or strong ligand-field splitting $(\lambda \ll \Delta) \overline{\delta \gamma^{2}}=\overline{\delta g^{2}}$, and the spin follows the rotation of the molecular axis only very loosely. For strong spin-orbit coupling or weak ligand-field splitting $(\lambda \gg \Delta)$, in linear radicals $\overline{\delta \gamma^{2}}$ approaches a value of 2 . In that case, Equation (10) no longer holds, but $\tau_{\mathrm{s}}$ approaches a limiting value of $\tau_{\mathrm{c}}{ }^{[64]}$

In case of the gta spin relaxation mechanism, there are different expressions for $T_{1}$ and $T_{2}$ [Eqs. (13) and (14)]:[65]

$$
\begin{aligned}
& \frac{1}{T_{1}}=\frac{1}{30}\left(g_{\|}-g_{\perp}\right)^{2} \frac{\omega_{0}^{2} \tau_{c}}{1+\omega_{0}^{2} \tau_{c}^{2}} \\
& \frac{1}{T_{2}}=\frac{1}{45}\left(g_{\|}-g_{\perp}\right)^{2} \omega_{0}^{2} \tau_{c}+\frac{1}{60}\left(g_{\|}-g_{\perp}\right)^{2} \frac{\omega_{0}^{2} \tau_{c}}{1+\omega_{0}^{2} \tau_{c}^{2}}
\end{aligned}
$$

We can assess the contributions of the various mechanisms in a semiquantitative manner using Equations (10)-(12) if we can make reasonable estimates of the orientational relaxation times $\tau_{c}$. We will base these estimates for the $\mathrm{NO}$ and $\mathrm{O}_{2}{ }^{--}$radicals on a comparison to suitable reference molecules rather than on an attempt to calculate the factor $f$ in Equation (12) from first principles. For $\mathrm{CS}_{2}$, which we use as reference for $\mathrm{NO}_{1}$ an experimental $\tau_{c}$ value of $1.4 \mathrm{ps}$ was determined in neat $\mathrm{CS}_{2}$ at $20^{\circ} \mathrm{C}(\eta=0.36 \mathrm{cP}) .^{[6]}$ It was shown by $\mathrm{Hu}$ and $\mathrm{Zwanzig}^{[62]}$ that this value is in correspondence with a value of $f$ obtained under the assumption of perfect slip conditions, while it would have been about $7.2 \mathrm{ps}$ if perfect stick conditions had applied. For NO in aqueous solution the viscosity is higher $(\eta=$ $1.002 \mathrm{CP}$ ), and the molecule is smaller and less prolate than $\mathrm{CS}_{2}$, but slip conditions between this molecule and the solvent may not be perfect. We therefore assume that for NO in water we should use a similar value as for $\mathrm{CS}_{2}$ in $\mathrm{CS}_{2}$, that is, $\tau_{\mathrm{c}}=1.4 \mathrm{ps}$.

The interaction of $\mathrm{O}_{2}{ }^{--}$with the solvent water is stronger than that of NO. As far as the size of the molecule and its interaction with surrounding water is concerned, we may well compare it with the interaction of a $\mathrm{H}_{2} \mathrm{O}$ molecule with surrounding $\mathrm{D}_{2} \mathrm{O}$, a situation for which $\tau_{\mathrm{c}}$ of the water molecules has been determined as $2.5 \mathrm{ps}^{\left[{ }^{[67]}\right.}$ Therefore, we use this as a value for $\mathrm{O}_{2}{ }^{--}$, too.

Before considering the possible range of relaxation rates to be expected for $\mathrm{NO}$ and $\mathrm{O}_{2}{ }^{--}$based on the mechanisms outlined above, we briefly mention the idea of another mechanism of spin relaxation for linear radicals in solution, sketched by Brocklehurst for the ' $\mathrm{OH}$ radical, but in principle also applicable to the $\mathrm{O}_{2}{ }^{--}$radical. ${ }^{[68]}$ The basic idea of this mechanism was already suggested for symmetric aromatic radical anions by McConnell in $1961{ }^{[69]}$ Phrased for the case of the $\mathrm{OH}$ radical, it is based on the idea that eventual sudden collapses of ligand-field splitting due to ruptures of hydrogen bonds will, for short times, create situations in which the spin will suddenly feel strong spin-orbit coupling and, since due to the sudden perturbation it is not in an eigenstate of the current spin Hamiltonian, will precess around the molecular axis. For a spinorbit coupling constant on the order of $100 \mathrm{~cm}^{-1}$, complete spin reorientation would be achieved in about $0.1 \mathrm{ps}$. To assess the real contribution of this mechanism to spin relaxation one would have to specify the frequency of such ligand field jumps and the time span available for free spin evolution under the spin-orbit coupling Hamiltonian. Although it is conceivable that the spin relaxation contribution of this mechanism may be substantial, to the best of our knowledge, this theory has not been developed to a more quantitative level. Moreover, the applicability of the quantum theoretical sudden-perturbation case, as invoked in that mechanism, cannot be taken for granted, considering that the splitting between the lowest two Kramers doublets (cf. Figure 7) varies between several 1000 and $100 \mathrm{~cm}^{-1}$, while the average thermal energy at room temperature corresponds to about $200 \mathrm{~cm}^{-1}$. If the perturbation due to the dynamic change of the ligand field is treated as adiabatic, the effect on the spin may become much smaller, unless rotation of the ligand field is involved, which brings us back to the ARES interpretation ${ }^{[63-64]}$ of spin-rotational interaction. In any case, the approach chosen in this work can be considered as a reasonable basis for estimating the correct order of magnitude of the rate of spin relaxation in a $\mathrm{NO} / \mathrm{O}_{2}{ }^{--}$radical pair.

In Figure 8, the contributions of the various spin relaxation mechanisms are plotted as functions of the magnetic field. In calculating these functions, the $g$-tensor components were derived from Equations (8) and (9). Within that $g$-tensor model they depend on just one parameter $x=\lambda / \Delta$. The spin-orbit coupling constant may be considered as independent of the medium, but this is not the case for the ligand-field splitting parameter $\Delta$. Although some information on this value for NO and $\mathrm{O}_{2}^{-}$is available for solid low-temperature matrices from the measured $g$-tensor values, it is unknown which values should be used in liquid aqueous solution at room temperature. Therefore, we consider different orders of magnitude for $x$. As representative values we chose $x= \pm 0.1$ (the negative sign referring to the $\mathrm{O}_{2}{ }^{--}$case), values that roughly describe the findings in the cryogenic hosts, and $x= \pm 1$ corresponding to a strongly reduced ligand-field splitting, as may be conceivable for the situation at room temperature in liquid solution, where the solvation shell of the radicals is dynamic and less or- 

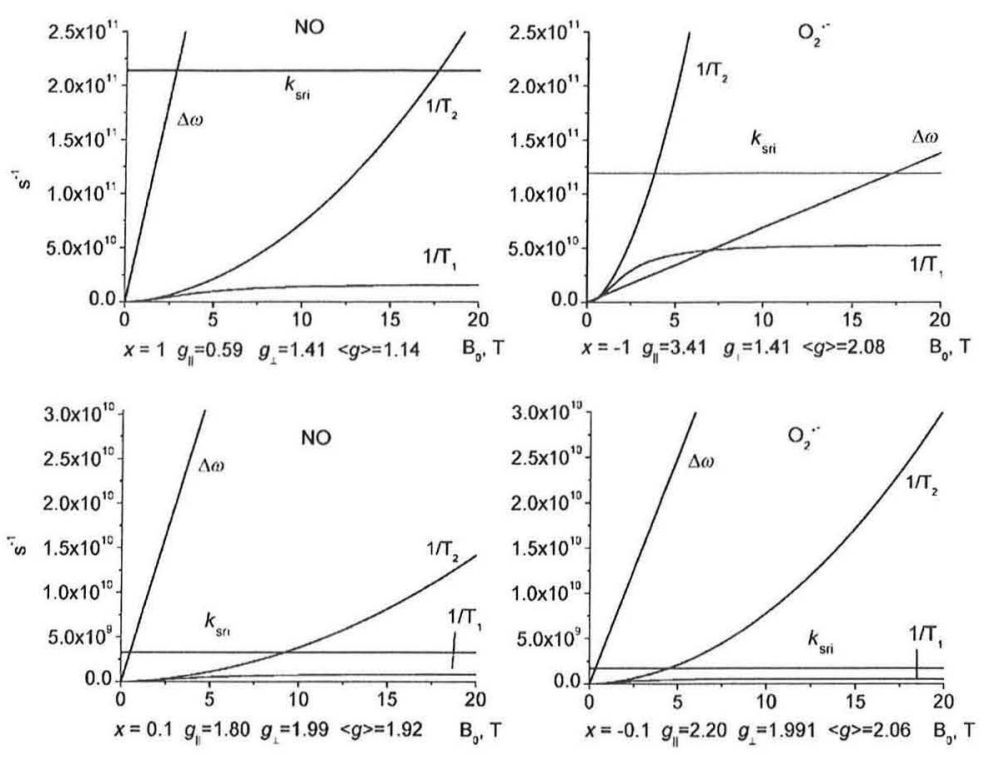

Figure 8. Comparison of field dependences of relaxation rate constants and $S / T_{0}$ mixing frequency. $k_{\text {sri }}$ : spin-rotational relaxation $\left(1 / T_{1}=1 / T_{2}\right), 1 / T_{1}$ for gta mechanism, $1 / T_{2}$ for gta mechanism, $\Delta \omega$ : angular frequency of $S / T_{0}$ mixing according to the $\Delta g$ mechanism assuming a "normal" counter-radical of the radical considered.

dered. It may be surmised, though, that the ligand field remains much stronger for the $\mathrm{O}_{2}^{--}$radical ion than for the neutral NO radical.

Apart from the curves for $\left(1 / T_{1,2}\right)_{\text {sri }}\left(1 / T_{1}\right)_{\text {gtar }}$ and $\left(1 / T_{2}\right)_{\text {gta }}$ the diagrams in Figure 8 also show the magnetic-field dependence of the $\Delta g$-type coupling $\Delta \omega$ [cf. Eq. (6)] between the $S$ and $\mathrm{T}_{0}$ states of the radical pair. For these calculations, $\Delta g$ was approximated as $\Delta g=\langle g\rangle-g_{\mathrm{e}}$ where $\langle g\rangle$ represents the isotropic $g$ value of the radical considered, and it was assumed that it is paired with a counter-radical for which spin-orbit coupling effects on the $g$ value are negligible.

For $x= \pm 0.1$, the pictures for $\mathrm{NO}$ and $\mathrm{O}_{2}{ }^{--}$are very similar. Over the full field range, $T_{1}$-type spin relaxation is dominated by the sri mechanism. Regarding the $T_{2}$ process, the gta contribution surpasses that of sri relaxation above 5-10 T. However $\left(1 / T_{2}\right)_{\text {gta }}$ always remains far below $\Delta \omega$ of the coherent $S / T_{0}$ transitions.

At higher values of $x$, that is, smaller ligand-field splitting, the situations for $\mathrm{NO}$ and $\mathrm{O}_{2}{ }^{--}$are quite different. This is because as the absolute value of $x$ increases, $g_{\|}$and $g_{\perp}$ move away from $g_{\mathrm{e}}$, both for positive (NO) and negative $\left(\mathrm{O}_{2}{ }^{--}\right)$values of $x$, but they both get smaller for NO, and thus reduce the value of $\langle g\rangle$, whereas for $\mathrm{O}_{2}^{--} g_{\|}$gets larger and $g_{\perp}$ smaller than $g_{\mathrm{e}}$ so that their effects are compensated when taking the average $\langle g\rangle$, and this quantity changes only little. In consequence, $\left(1 / T_{1,2}\right)_{\text {sri }}$ increases strongly both for $\mathrm{NO}$ and $\mathrm{O}_{2}{ }^{--}$. The same is true for $\left(1 / T_{1}\right)_{\text {gta }}$, which, however, increases more strongly for $\mathrm{O}_{2}^{--}$than for $\mathrm{NO}$, but even in the latter case stays clearly below $\left(1 / T_{1,2}\right)_{\text {sri }}$ up to $20 \mathrm{~T}$. Such a quantitative difference between $\mathrm{NO}$ and $\mathrm{O}_{2}^{-}$is also seen in the curves for $\left(1 / T_{2}\right)_{\text {gta- }}$. This value increases more strongly for $\mathrm{O}_{2}{ }^{--}$, surpassing the value of $\left(1 / T_{1,2}\right)_{\text {sri }}$ at about $4 \mathrm{~T}$, while in the case of NO this happens only at $18 \mathrm{~T}$.
An interesting consequence follows from the different behavior of $\langle g\rangle$ in the two radicals. Whereas for NO $\Delta \omega$ is much larger than $\left(1 / T_{2}\right)_{\text {gta }}$ at all fields, the opposite holds in the case of $\mathrm{O}_{2}^{--}$. This means that in case of a radical pair made up of $\mathrm{O}_{2}{ }^{--}$and a "normal" radical (i.e., with $<g>\approx g_{\mathrm{e}}$ and $g_{\perp} \approx g_{\|}$) the mechanism of $\mathrm{S} / \mathrm{T}_{0}$ transitions would be dominated by the incoherent phase relaxation in $\mathrm{O}_{2}{ }^{-}$rather than by the coherent $\mathrm{S} / \mathrm{T}_{0}$ mixing due to the difference in Larmor frequencies $\Delta \omega$. However, in view of the supposedly larger ligand-field splitting of $\mathrm{O}_{2}^{--}$in water, it is assumed in the following model calculations on the MFE that the properties of NO will dominate the spin dynamics, whereas $\mathrm{O}_{2}{ }^{\circ}$ will be assumed to behave like a "normal" radical.

\subsection{Modeling the Kinetic MFE on Radical Recombination}

As was pointed out in the Introduction and at the beginning of the Discussion, it is reasonable to assume that the MFE on the recombination rate of $\mathrm{NO}$ and $\mathrm{O}_{2}^{-{ }^{-}}$(or of one of these with some other radical) is due to the Zeeman effect of the external magnetic field on the two radical spins, which induces triplet/ singlet transitions in the pair during their encounter and thus allows to some extent recombination of radicals that enter an encounter with initial triplet spin. ${ }^{[70]}$ As noted when explaining Scheme 1, the radical recombination is predominantly between radicals created from different SIN-1 precursor molecules, such that no a priori spin correlation exists between them (so-called F-pairs). ${ }^{[71]}$ Quantitative theories to deal with such situations have been provided by Mints and Pukhov ${ }^{[72]}$ and by Gorelik et al. ${ }^{[73]}$ The treatment by the latter is based on integral encounter theory ${ }^{[74]}$ and is more general. Thus we base our calculations on the equations given there (for details, see the Supporting Information).

According to reference [73], the recombination reaction is described in terms of Scheme 2 adapted to our reaction

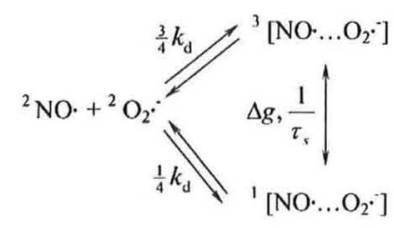

Scheme 2. Kinetic scheme characterising the spin-chemical assumptions underlying the theoretical treatment. (Adapted from ref. [73].) 
system. The radical pairs are formed at diffusion controlled rate modified by a spin statistical factor of $1 / 4$ for each of the 4 spin substates. The rate constant $k_{\mathrm{d}}$ is given by Equation (15):

$k_{\mathrm{d}}=4 \pi R D N_{\mathrm{A}}$

where $R$ is the reaction distance, $D$ is the sum of the diffusion coefficients of the two radical species, and $N_{\mathrm{A}}$ is Avogadro's constant. The reaction is assumed to occur in a reaction zone of thickness $\delta$ wherein its rate constant is assigned a value of $W_{0}$. This quantity specified, the bulk second-order rate constant $k_{r}$ of radical recombination under conditions where diffusion is infinitely fast would correspond to Equation (16):

$k_{r}=4 \pi R^{2} \delta W_{0} N_{A}$

It is important to distinguish the (not directly observable, virtual) reaction rate constant $k_{n}$ which we call "true" reaction rate constant, from the observable rate constant $k_{\text {rec }}$, which we call "apparent" rate constant. If no spin conversion takes place during the encounter of a radical pair only those radical pairs encountering with singlet spin could react. In that case the apparent second-order rate constant $k_{\text {rec }}$ would be given by Equation (17): ${ }^{[73]}$

$k_{\text {rec }}^{0}=\frac{1}{4} \frac{k_{\mathrm{d}} k_{\mathrm{r}}}{k_{\mathrm{d}}+k_{\mathrm{r}}}$

In reality, radical-pair encounters with triplet spin can also lead to recombination if during the period of geminate re-encounters the multiplicity changes, a process that can occur through spin relaxation in the individual radicals and/or coherent motion of the electron spins in the external magnetic field. ${ }^{[75]}$ To estimate the maximum effect a magnetic field can exhibit on $k_{\text {rec }}$ one could assume that the field would make the conversion between $S$ and $T_{0}$ infinitely fast. In that case these two spin substates would behave as two states each with $50 \%$ singlet character. Accordingly, Equation (17) would change to Equation (18):

$k_{\mathrm{rec}}^{\infty}=\frac{1}{2} \frac{k_{\mathrm{d}} \frac{k_{\mathrm{r}}}{2}}{k_{\mathrm{d}}+\frac{k_{\mathrm{r}}}{2}}=\frac{1}{4} \frac{k_{\mathrm{d}} k_{\mathrm{r}}}{k_{\mathrm{d}}+\frac{k_{\mathrm{r}}}{2}}$

Hence, the limiting high-field MFE $\varphi_{k_{\text {rec }}}^{\infty}$ on $k_{\text {rec }}$ would be given by Equation (19):

$\varphi_{k_{\text {rec }}}^{\infty}=\frac{k_{\text {rec }}^{\infty}-k_{\mathrm{rec}}^{0}}{k_{\mathrm{rec}}^{0}}=\frac{1}{1+2 \frac{k_{\mathrm{d}}}{k_{\mathrm{r}}}}<1$

It depends on the $k_{\mathrm{d}} / k_{\mathrm{r}}$ ratio and it could reach a value as high as $100 \%$ in the limit $k_{\mathrm{r}} \gg k_{\mathrm{d}}$. In principle, the increase of $k_{\text {rec }}$ with increasing rate of $\mathrm{T}_{0} / \mathrm{S}$ transitions is due to the fact that it is more efficient to distribute the reactivity over more than one state, even though the reaction rate constant for each of these states is reduced in proportionality to its singlet character. In reality, the individual spin relaxation processes of the two radicals contribute to the rate of $\mathrm{T}_{0} / \mathrm{S}$ (and $\mathrm{T}_{ \pm} / \mathrm{S}$ ) tran- sitions. Therefore, $k_{\text {rec }}$ in zero field is already larger than $k_{\text {rec }}^{0}$. Furthermore, at finite fields the field-induced $T_{0} / S$ process is of finite rate. Therefore, $k_{\text {rec }}\left(B_{0}\right)$ is always smaller than $k_{\text {rec }}^{\infty}$ and the value of $\varphi_{k_{\text {rec }}}^{\infty}$ is always smaller than that given in Equation (19).

In the theoretical papers mentioned, ${ }^{[72,73]}$ the kinetic problem is solved by taking explicitly into account diffusion with geminate re-encounters, coherent $T_{0} / S$ mixing by the external magnetic field according to the $\Delta g$ mechanism, and incoherent $\mathrm{T}_{ \pm} \rightleftarrows \mathrm{T}_{0} \rightleftarrows \mathrm{S} \rightleftarrows \mathrm{T}_{ \pm}$transitions according to individual spin relaxation in the two radicals. In ref. [73] the simplification is made that spin relaxation in only one of the radicals is relevant and that transversal and longitudinal relaxation times are equal, that is, $T_{1}=T_{2}$. For the purpose of a semiquantitative estimation this approximation is not a serious one. In the discussion above, arguments were provided that it is the NO radical that carries most of these properties and in comparison to which $\mathrm{O}_{2}^{--}$might be treated as a "normal" radical.

In reference [73], an analytical, albeit quite complex, expression for the dependence of $k_{\text {rec }}$ on the parameters of the model is given (see the Supporting Information) [Eq. (20)]:

$k_{\text {rec }}=k_{\text {rec }}\left(\Delta g \cdot B_{0}, \tau_{\mathrm{s}}, k_{\mathrm{r}}\right)_{\delta, R, D}$

In our model calculation, the parameters $\delta, R$, and $D$ are kept fixed. For $D$ we use a value of $3.4 \times 10^{-5} \mathrm{~cm}^{2} \mathrm{~s}^{-1}$ and for $R$ a value of $3.65 \AA$, as given by Bott et al. ${ }^{[40]}$ For $\sigma$-bond formation, a reaction zone $\delta$ of $0.3 \AA$ seems physically reasonable, although the absolute value of this parameter is not important as long as it is small and the reaction rate constant $W_{0}$ in this zone [and hence $k_{n}$ cf. Eq. (16)] is a free fitting parameter. ${ }^{[76]}$ Two characteristic times derived from the parameter values given here are $\tau_{\mathrm{d}}$, the average time of diffusional separation of a radical pair, and $\tau_{\mathrm{e}}$ the dwell time of the radical pair in the reaction zone [Eqs. (21) and (22)]:

$\tau_{\mathrm{d}}=\frac{R^{2}}{D}=39 \mathrm{ps}$

$\tau_{\mathrm{e}}=\frac{R \delta}{D}=3.2 \mathrm{ps}$

The value of the orientational relaxation time $\tau_{\mathrm{c}}=1.4 \mathrm{ps}$ was already given above.

To provide an idea of the parameter dependence of the MFE on $k_{\text {rec }}$ in Figure 9 we show characteristic curves for this MFE as a function of the "effective field" $\Delta g B_{0}$ bringing about the coherent transitions between $T_{0}$ and $\mathrm{S}$. The different curves refer to a variation of the spin relaxation time $\tau_{s}$. The MFE strongly increases as $\tau_{\mathrm{s}}$ gets longer. At the same time, the main rise of the MFE is shifted to lower fields. For $\tau_{s} \rightarrow \infty$, the limiting MFE at infinite field reaches the value that is predicted by Equation (19) (see Table 1). An interesting point is the curvature of the MFE in the region from 0 to $20 \mathrm{~T}$. A rough measure of the concavity of the curves is given by the ratio of the MFE at 20 and $10 \mathrm{~T}$. A value of 2 would be expected for linear behavior, while values greater than 2 indicate upward concave curvature, and values less than 2 downward concave curvature. It follows from Table 1 that the curvature behavior changes be- 


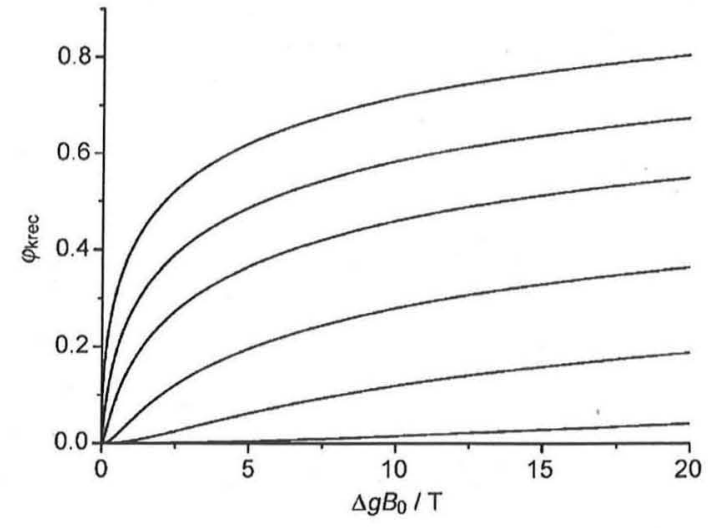

Figure 9. Magnetic-field effect on $k_{\text {rec }}$ as a function of the effective magnetic field $\Delta g B_{0}$ for various values of spin relaxation time $\tau_{s}$ (from bottom): 1,5 , $20,100,500$, and $10^{9} \mathrm{ps}$. Values of fixed parameters: $k_{\mathrm{r}}=5 \times 10^{11} \mathrm{M}^{-1} \mathrm{~s}^{-1}$, $D=3.4 \times 10^{-5} \mathrm{~cm}^{2} \mathrm{~s}^{-1}, R=3.65 \AA, \delta=0.3 \AA$.

\begin{tabular}{|c|c|c|c|}
\hline$\tau_{\mathrm{c}}[\mathrm{ps}]$ & $\varphi_{k_{\text {tec }}}(20)[\%]$ & $\varphi_{k_{\text {tec }}}(\infty)[\%]$ & $\frac{\varphi_{k_{\text {tex }}}(20)}{\varphi_{k_{\text {tec }}}(10)}$ \\
\hline 1 & 4.1 & 16.30 & 2.67 \\
\hline 5 & 18.7 & 34.62 & 1.57 \\
\hline 20 & 36.4 & 53.30 & 1.30 \\
\hline 100 & 54.9 & 71.88 & 1.19 \\
\hline $10^{9}$ & 80.3 & $96.39^{\text {(a) }}$ & 1.12 \\
\hline
\end{tabular}

[a] Agrees with the limiting value of $\varphi_{k_{\text {tex }}}(\infty)$ according to Equation (19): $96.39 \%$

tween $\tau_{\mathrm{c}}=1$ and $5 \mathrm{ps}$. This point will be of some significance when comparing the theoretical simulations with the observed MFE.

In Figure 9, some general predictions of the spin chemical model of $\mathrm{NO} / \mathrm{O}_{2}^{--}$recombination specified by Scheme 1 are presented. To sharpen the focus on this concrete system, we need to employ the specific magnetic properties of these radicals, as discussed above. Using Equations (8)-(14), we can express the essential spin-chemical parameters $\tau_{\mathrm{s}}$ and $\Delta g$ as functions of just one parameter $x=\lambda / \Delta$, the ratio of spin-orbit coupling constant and ligand-field splitting in the NO radical.

When investigating the dependence of the MFE $\varphi_{k_{\text {rec }}}$ on the parameter $x$, it is found that it steeply rises with $x$ up to a maximum around $x \approx 0.2$, after which it decreases slowly. This finding is rather independent of the reaction rate constant $k_{r}$ over a wide range of the latter parameter (see Figure S3, Supporting Information). On the other hand, for $x \approx 0.2$, the curvature of the magnetic-field dependence of $\varphi_{k_{r a c}}$ is rather pronounced [curvature parameter $c=\varphi_{\mathrm{k}_{\text {rec }}}(20 \mathrm{~T}) / \varphi_{\mathrm{k}_{\mathrm{rec}}}(10 \mathrm{~T}) \approx 1.45$ ] and gets considerably less $(c \rightarrow 1.75)$ if $x$ is allowed to reach a value of 1 (Figure S4, Supporting Information). In Figure 10 we show the predicted field dependence of the MFE $\varphi_{k_{\text {rec }}}$ for $x=1$ and for several values of the true reaction rate constant $k_{r}$ Interestingly, the curves obtained do not vary in a monotonic way with $k_{r}$ The MFE $\varphi_{k_{\text {rec }}}$ at $20 \mathrm{~T}$ passes through a maximum at $k_{r}$ $\approx 120 \times 10^{9} \mathrm{M}^{-1} \mathrm{~s}^{-1}$. At this point also the curvature (see Table 2) starts to decrease and continues to do so up to $k_{\mathrm{r}} \approx 1000 \times$

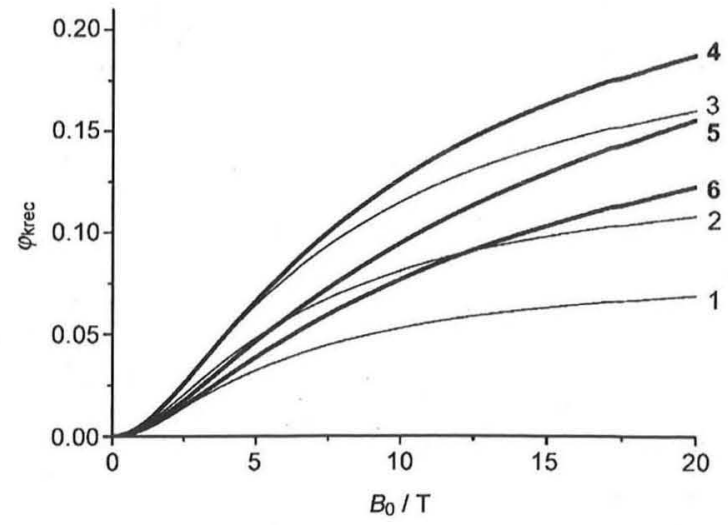

Figure 10. Magnetic-field effect on $k_{\text {rec }}$ as a function of the magnetic field for various values of the reaction rate constant $k_{\mathrm{r}}$ (in units of $\mathrm{M}^{-1} \mathrm{~s}^{-1}$ ): 1) $10^{10}$, 2) $2 \times 10^{10}$, 3) $5 \times 10^{10}$, 4) $1.2 \times 10^{11}$, 5) $10^{12}$, 6) $10^{14}$. All spin parameters were derived from Equations ( 8 ) $-(12)$ by using $\lambda / \Delta=1$. For the values of the other parameters, see Table 2 and Figure 9.

\begin{tabular}{|c|c|c|c|c|}
\hline$k_{\mathrm{r}}\left[10^{9} \mathrm{M}^{-1} \mathrm{~s}^{-1}\right]$ & $k_{\text {rec }}\left[10^{9} \mathrm{M}^{-1} \mathrm{~s}^{-1}\right]$ & $\varphi_{k_{\text {tee }}}(20)[\%]$ & $\varphi_{k_{\text {tee }}}(\infty)[\%]$ & $\frac{\varphi_{k_{\text {tec }}}(20)}{\varphi_{k_{\text {rec }}}(10)}$ \\
\hline 10 & 1.7 & 6.6 & 8.8 & 1.32 \\
\hline 20 & 2.5 & 10.4 & 14.1 & 1.35 \\
\hline 50 & 3.6 & 15.4 & 22.2 & 1.42 \\
\hline 120 & 4.3 & 18.0 & 28.5 & 1.51 \\
\hline $10^{3}$ & 4.9 & 14.8 & 34.7 & 1.68 \\
\hline$\infty$ & 5.0 & 11.6 & 35.8 & 1.63 \\
\hline
\end{tabular}

[a] The values of the spin parameters employed $\left(\tau_{\mathrm{s}}=4.6 \mathrm{ps}, \overline{\delta g^{2}}=2.69\right.$, $\Delta g=0.86$ ) were derived from Equations (8)-(12) by using $\lambda / \Delta=1$.

$10^{9} \mathrm{M}^{-1} \mathrm{~s}^{-1}$. Note, however, that in the limit of very high field, $\varphi_{k_{\text {rec }}}$ does increase monotonically with $k_{r}$ (see Table 2 ).

The rationale for an increase of the MFE $\varphi_{k_{\text {rec }}}$ was given in the discussion of Equation (19). It is strictly valid in the limit of infinitely high magnetic field. At finite field, however, increasing $k_{r}$ is tantamount to introducing a lifetime broadening of the RP singlet state. This leads to a slowing down of the $S / T_{0}$ conversion process for a given coupling strength $\left(\triangle g B_{0}\right)$, but can be overcome if the coupling strength is increased correspondingly. ${ }^{[77]}$ From a comparison of the curves in Figure 10 with the size and the apparently linear behavior of the experimental MFE $\varphi_{Y}$ shown in Figure 6, we conclude that a $k_{r}$ value on the order of $(200-1000) \times 10^{9}$ would be suitable to account for the observed effects. (For a connection between the theoretical MFEs on $k_{\text {rec }}$ and the observed MFE on the yield of $\mathrm{ONOO}^{-}$, see below.) While such an order of magnitude for $k_{\mathrm{r}}$ seems very high, it does not lead to unrealistically high values for $k_{\text {rec }}$ (cf. Table 2), which, however, is a matter of course, since $k_{\text {rec }}$ is bounded by $k_{\mathrm{d}}$. When discussing the relevance of the $k_{\mathrm{r}}$ value, one must convert it to $W_{0}[\mathrm{Eq} .(16)]$, the first-order rate constant in the reaction zone. For $k_{r}=200 \times 10^{9} \mathrm{M}^{-1} \mathrm{~s}^{-1}$, we obtain $W_{0}=10^{13} \mathrm{~s}^{-1}$ corresponding to a vibrational frequency of about $300 \mathrm{~cm}^{-1}$. It seems reasonable to assume that formation of the $\mathrm{ON}-\mathrm{OO}^{-}$bond is activationless, and sliding down 
the bonding potential surface might well occur on a timescale comparable to a low-frequency molecular vibration.

\subsection{Modeling the MFE on the Product Yield}

The last step of a theoretical simulation of the MFE on the reaction yield $Y$ of the recombination product $\mathrm{ONOO}^{-}$is to relate the values of $k_{\text {rec }}$ and $Y$. Although all the details of side reactions of $\mathrm{NO}$ and $\mathrm{O}_{2}{ }^{--}$are not precisely known, or too complex in their entity, it is clear that such reactions must exist because a MFE on the yield $Y$ can follow from a MFE on $k_{\text {rec }}$ only if the yield is less than $100 \%$, that is, only if there is competition between radical recombination to form $\mathrm{ONOO}^{-}$(and from it $\mathrm{RH}$ ) and some other "sink channels" of $\mathrm{NO}$ and $\mathrm{O}_{2}^{-{ }^{-}}$that are less sensitive to a magnetic field.

To see how the MFEs on $k_{\text {rec }}$ are mapped to the MFEs on $Y$, it is useful to consider the mathematical relation between the two kinds of MFEs in a rather general way. The total magneticfield effect on the yield can be expressed as an integral over the differential magnetic-field effect on the yield [Eq. (23)]:

$\varphi_{Y}=\frac{1}{Y_{0}} \int_{0}^{B_{0}} \frac{\mathrm{d} Y}{\mathrm{~d} B_{0}} \mathrm{~d} B_{0}$

Taking into account the dependence of $Y$ on the kinetic parameters we can expand this as Equation (24):

$\varphi_{Y}=\frac{1}{Y_{0}} \int_{0}^{B_{0}}\left(\frac{\partial Y}{\partial k_{\text {rec }}}\right) \frac{\mathrm{d} k_{\text {rec }}}{d B_{0}} \mathrm{~d} B_{0}$

To first order we may assume that the dependence of $Y$ on $k_{\text {rec }}$ does not depend on the magnetic field and hence rewrite Equation (23) as Equation (25):

$\varphi_{Y} \approx \frac{1}{Y_{0}}\left(\frac{\partial Y}{\partial k_{\mathrm{rec}}}\right) \int_{0}^{B_{0}} \frac{\mathrm{d} k_{\mathrm{rec}}}{\mathrm{d} B_{0}} \mathrm{~d} B_{0}$

which can be rewritten as Equation (26):

$$
\begin{aligned}
\varphi_{Y} & \approx \frac{1}{Y_{0}}\left(\frac{\partial Y}{\partial k_{\mathrm{rec}}}\right) \frac{k_{\mathrm{rec}, 0}}{k_{\mathrm{rec}, 0}} \int_{0}^{B_{0}} \frac{\mathrm{d} k_{\mathrm{rec}}}{\mathrm{d} B_{0}} \mathrm{~d} B_{0} \\
& =\frac{k_{\mathrm{rec}, 0}}{Y_{0}}\left(\frac{\partial Y}{\partial k_{\mathrm{rec}}}\right)_{0} \varphi_{k_{\mathrm{rec}}}
\end{aligned}
$$

This means that there is a direct proportionality between $\varphi_{Y}$ and $\varphi_{k_{\text {rec }}}$ the two types of magnetic-field effects, with the proportionality constant $q$ between them given by Equation (27):

$q=\frac{k_{\mathrm{rec}, 0}}{Y_{0}}\left(\frac{\partial Y}{\partial k_{\mathrm{rec}}}\right)_{0}$
To establish some general relations between the constant $q$ and the yield $Y$ we consider the following generic kinetic situations.

1) The sink channels for $\mathrm{NO}$ and $\mathrm{O}_{2}{ }^{--}$are assumed to be both of first-order kinetics. In this case the following rate equations hold [Eqs. (28) and (29)]:

$$
\begin{aligned}
& \frac{\mathrm{d}[\mathrm{NO}]}{\mathrm{d} t}=k_{2}[\mathrm{SIN}-1 \mathrm{~A}]-k_{\mathrm{rec}}[\mathrm{NO}]\left[\mathrm{O}_{2}^{-}\right]-k_{1}^{(1)}[\mathrm{NO}] \\
& \frac{\mathrm{d}\left[\mathrm{O}_{2}^{-}\right]}{\mathrm{d} t}=k_{2}[\mathrm{SIN}-1 \mathrm{~A}]-k_{\mathrm{rec}}[\mathrm{NO}]\left[\mathrm{O}_{2}^{-}\right]-k_{2}^{(1)}\left[\mathrm{O}_{2}^{-}\right]
\end{aligned}
$$

There is no analytical solution for the product yield as a function of the rate constants in this situation. So the system of differential equations was integrated numerically in order to determine $Y$ as a function of $k_{1}^{(1)}$ and $k_{2}^{(1)}$ for fixed values of all the parameters determining $k_{\text {rec }}$.

2) The sink channels for $\mathrm{NO}$ and $\mathrm{O}_{2}{ }^{-}$are assumed to be both of second-order kinetics with the following rate equations [Eqs. (30) and (31)]:

$$
\begin{aligned}
& \frac{\mathrm{d}[\mathrm{NO}]}{\mathrm{d} t}=k_{2}[\mathrm{SIN}-1 \mathrm{~A}]-k_{\mathrm{rec}}[\mathrm{NO}]\left[\mathrm{O}_{2}^{-}\right]-k_{1}^{(2)}[\mathrm{NO}]^{2} \\
& \frac{\mathrm{d}\left[\mathrm{O}_{2}^{-}\right]}{\mathrm{d} t}=k_{2}[\mathrm{SIN}-1 \mathrm{~A}]-k_{\mathrm{rec}}[\mathrm{NO}]\left[\mathrm{O}_{2}^{-}\right]-k_{2}^{(2)}\left[\mathrm{O}_{2}^{-}\right]^{2}
\end{aligned}
$$

For this case it can be shown (see the Supporting Information) that the yield $Y_{\mathrm{ONOO}^{-}}$of $\mathrm{ONOO}^{-}$is independent of $k_{2}$ determining the rate of formation of the radicals, and it is given by Equation (32):

$Y_{\mathrm{ONOO}^{-}}=\frac{k_{\mathrm{rec}}}{k_{\mathrm{rec}}+\sqrt{k_{1}^{(2)} k_{2}^{(2)}}}$

We will assume that the yield $Y$ of $\mathrm{RH}$ will be directly proportional to $Y_{\mathrm{ONOO}^{-}}$and not depend on a magnetic field, so that the MFE on both will be equal.

From Equations (27) and (32) we can directly calculate the factor $q$ relating $\varphi_{Y}$ and $\varphi_{k_{\text {rec }}}$. The result is Equation (33):

$q=\frac{\sqrt{k_{1}^{(2)} k_{2}^{(2)}}}{k_{\mathrm{rec}}+\sqrt{k_{1}^{(2)} k_{2}^{(2)}}}=1-Y$

So, in this case, the factor $q$ and the yield $Y$ are directly related in a linear fashion, independent of the individual values of $k_{1}^{(2)}$ and $k_{2}^{(2)}$.

3) Case of mixed first- and second-order sink channels: In this case, too, numerical integration had to be employed.

The results of our calculations of the factor $q$ are plotted against the values of the product yield $Y$ for large variations of $k_{1}^{(1)}, k_{2}^{(1)}, k_{1}^{(2)}$, and $k_{2}^{(2)}$ in Figure 11 for cases 1)-3). Not only in case 2), where this was derived analytically, but also in the other two cases the results fall on single lines, that is, in all cases the value of $q$ is uniquely determined by the value of $Y$, independent of the individual values of $k_{1}^{(1)}$ and $k_{2}^{(1)}$ in case 1, 


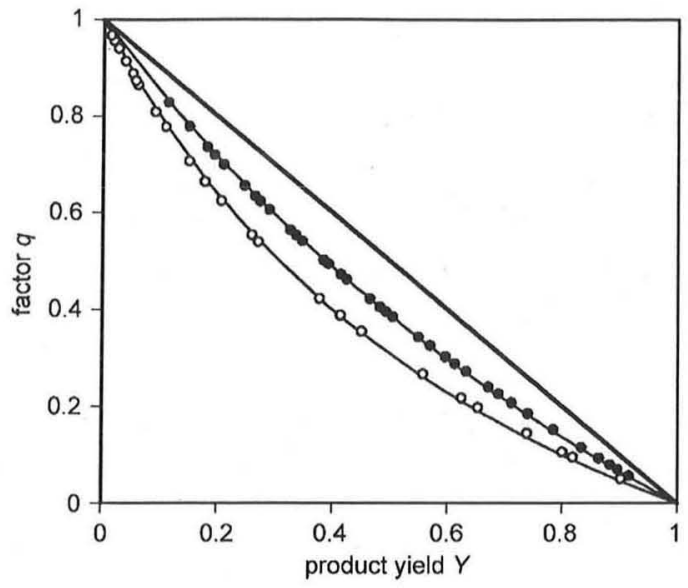

Figure 11. Dependence of factor $q$ on the product yield. Solid straight line $(-)$ : case of second-order sink channels. Open circles (o): case of firstorder sink channels. Closed circles ( $\bullet$ ): case of mixed first- and second-order sink channels. The lines through the data points represent fit curves following the empirical relation $q=b(1-\gamma) /(\gamma+b)$ with $b=0.80$ (first order) and 1.74 (mixed first and second order).

of $k_{1}^{(2)}$ and $k_{2}^{(2)}$ in case 2 , or $k_{1}^{(1)}$, and $k_{2}^{(2)}$ in case 3. For the constant set of all other rate parameters used, the relation between $q$ and $Y$ is not linear, but upwardly concave, that is, as $Y$ increases $q$ is always lower than in the linear case. The curve for the mixed-order sink channels runs between those of pure second-order and pure first-order.

We may briefly comment on the possible chemical nature of the sink channels. Assuming that the yield of $\mathrm{ONOO}^{-}$in the decomposition of SIN-1 is around 0.5, as will be substantiated below, our calculations show that it would be required that the geometric average of $k_{1}^{(2)}$, and $k_{2}^{(2)}$ be on the order of $5 \times$ $10^{9} \mathrm{M}^{-1} \mathrm{~s}^{-1}$ in the case of second-order kinetic sink channels, or the geometric average of $k_{1}^{(1)}$ and $k_{2}^{(1)}$ should be on the order of $2 \mathrm{~s}^{-1}$ in case of first-order (or pseudo-first-order) kinetic sink channels. According to the literature, the most prominent decay reaction of $\mathrm{O}_{2}{ }^{--}$is its disproportionation [Eq. (34)].:"78]

$\mathrm{HO}_{2}{ }^{-}+\mathrm{O}_{2}{ }^{--}+\mathrm{H}_{2} \mathrm{O} \rightarrow \mathrm{H}_{2} \mathrm{O}_{2}+\mathrm{O}_{2}+\mathrm{OH}^{-}$

with $k_{(34)}=9.7 \times 10^{7} \mathrm{M}^{-1} \mathrm{~s}^{-1}$. It involves the pre-equilibrium [Eq. (35)]:

$\mathrm{O}_{2}{ }^{--}+\mathrm{H}^{+} \rightleftharpoons \mathrm{HO}_{2} \cdot$

with an equilibrium constant $K_{(35)}=6.25 \times 10^{4}$. Assuming that the protolytic equilibrium is rapidly established, for $\mathrm{pH} 7.6$ one can deduce an effective second-order rate constant of $1.5 \times$ $10^{5} \mathrm{M}^{-1} \mathrm{~s}^{-1}$ for the disproportionation of $\mathrm{O}_{2}{ }^{--}$. This order of magnitude is far below the value required to decrease the recombination yield of $\mathrm{NO}$ and $\mathrm{O}_{2}{ }^{--}$appreciably.

For NO, one could discuss the third-order reaction [Eq. (36)]: ${ }^{[79]}$

$2 \mathrm{NO}+\mathrm{O}_{2} \rightarrow 2 \mathrm{NO}_{2}$ with $k_{(36)}^{(3)}=6.3 \times 10^{6} \mathrm{M}^{-2} \mathrm{~s}^{-1}$. For an $\mathrm{O}_{2}$ concentration of $280 \mu \mathrm{M}$ in air-saturated aqueous solution at room temperature, the reaction becomes pseudo-second-order with a rate constant of $k_{(36)}^{(2)}=1.8 \times 10^{2} \mathrm{M}^{-1} \mathrm{~s}^{-1}$. This value is far below that of a nearly diffusion controlled radical recombination reaction and therefore cannot decrease the recombination yield of $\mathrm{NO}$ and $\mathrm{O}_{2}{ }^{--}$.

So, for neither $\mathrm{NO}$ nor $\mathrm{O}_{2}^{--}$are there obvious candidates of second-order reactions to account for a reduced yield of $\mathrm{ONOO}^{-}$, necessary to explain a .MFE. On the other hand, if the required rate constant of $2 \mathrm{~s}^{-1}$ for a first-order kinetic sink channel is interpreted as a pseudo-first-order rate constant, reaction partners present in as low as $10^{-6}-10^{-5} \mathrm{M}$ concentration could be relevant if they react with these radicals at moderate rate, that is, with rate constants of $10^{5}-10^{6} \mathrm{M}^{-1} \mathrm{~s}^{-1}$. In this regard, even potential impurities of for example, $\mathrm{Fe}^{3+}$ ions present in one of the buffer components might have an effect. Thus, it is known that at $\mathrm{pH} 7.6$, EDTA complexes of $\mathrm{Fe}^{3+}$ react with $\mathrm{O}_{2}{ }^{--}$with a rate constant of $7 \times 10^{5} \mathrm{M}^{-1} \mathrm{~s}^{-1}$. ${ }^{[80]}$ According to the supplier's analysis, $\mathrm{Fe}$ is present in our p.a. quality $\mathrm{KH}_{2} \mathrm{PO}_{4}$ with a concentration of $\leq 0.001 \%$, which would amount to $\leq 1.6 \mu \mathrm{M}$ in the sample solutions. The DTPA complex of $\mathrm{Fe}^{3+}$ formed in our case would probably not be less reactive than the EDTA complex. However, at the present stage of investigation no further, specific information on the assignment of the active sink reactions for $\mathrm{NO}$ and $\mathrm{O}_{2}{ }^{--}$can be given. They will be explored during further work in progress.

\subsection{Comparison of the Theoretical MFE on $\boldsymbol{k}_{\text {rec }}$ with the Experimental MFE on the Yield of RH}

Figure 6 shows, together with the experimental data points of the MFE on the yield of $\mathrm{RH}$, three theoretical curves, calculated by assuming the standard values of the reaction parameters: $R=3.4 \AA, \delta=0.3 \AA, \tau_{\mathrm{c}}=1.4 \mathrm{ps}, x=1$, and $k_{\mathrm{r}}=2 \times 10^{11} \mathrm{~s}^{-1}$ (yielding a value of $k_{\text {rec }}=5.1 \times 10^{9} \mathrm{M}^{-1} \mathrm{~s}^{-1}$ ) and values of $0.25,0.31$, and 0.37 for $q$. These values would correspond to relative yields of $\mathrm{ONOO}^{-}$of $0.75,0.69$, and 0.63 in the case of secondorder sink channels, and $0.57,0.50$, and 0.43 , respectively, in the case of first-order sink channels. As can be seen, the theoretical curves fit the experimental data surprisingly well.

\subsection{Temperature Dependence of the MFE}

A preliminary experimental test of the temperature dependence of the MFE yielded a decrease of the value of $\varphi_{Y}=5.5 \%$ at $18 \mathrm{~T}$ to $\varphi_{Y}=3.1 \%$, that is, a relative reduction by $45 \%$, on increasing the temperature from $23^{\circ} \mathrm{C}$ to $40^{\circ} \mathrm{C}$. Qualitatively, a reduction of the MFE on $\varphi_{k_{\text {rec }}}$ is to be expected if, all other parameters being unchanged, the spin relaxation time of the radical pair decreases, because this increases the rate of T/S transitions independent of an external magnetic field, so that the field-induced contribution to this process will lose in relative weight.

Considering a more quantitative analysis of the temperature effect in the framework of the present theory, we must take into account a temperature-dependent change of solvent viscosity $\eta$ from $1.002 \mathrm{mPas}$ at $23^{\circ} \mathrm{C}$ to $0.653 \mathrm{mPas}$ at $40^{\circ} \mathrm{C}$, of 
the diffusion coefficient $D$ from $3.4 \times 10^{-5} \mathrm{~cm}^{2} \mathrm{~s}^{-1}$ to $5.5 \times$ $10^{-5} \mathrm{~cm}^{2} \mathrm{~s}^{-1}$, and of the spin relaxation time $\tau_{\mathrm{s}}$ from $4.67 \mathrm{ps}$ to $2.88 \mathrm{ps}$. Leaving all the other parameters constant with values used as a standard set (see caption of Figure 6), we calculate that $\varphi_{k_{\text {rec }}}$ is reduced by $25 \%$ on increasing the temperature from 23 to $40^{\circ} \mathrm{C}$. This is not quite as big as observed for $\varphi_{y}$ but of the right direction and order of magnitude. In view of the many parameters that might be optimized, a better agreement would be certainly fortuitous.

\section{Conclusions}

We have shown that there is a significant MFE on the yield of a reaction product, the formation of which involves recombination of two small inorganic radicals, $\mathrm{NO}$ and $\mathrm{O}_{2}{ }^{--}$. As was detailed in the theoretical analysis, the MFE shows all characteristics of a field-induced acceleration of the free (i.e., nongeminate) recombination of the two radicals. If this interpretation were confirmed, the effect would represent the first evidence of spin-chemical behavior of these radicals, which have escaped so far magnetic resonance detection in liquid solution. We are aware, though, that without more experimental evidence on the postulated reaction scheme, and in particular the chemical nature of the necessary sink channels for $\mathrm{NO}$ and $\mathrm{O}_{2}{ }^{--}$, the interpretation remains hypothetical.

The theoretical treatment of the spin-chemical kinetics of recombination of $\mathrm{NO}$ and $\mathrm{O}_{2}^{--}$was carried out in the framework of a simplified spin-chemical scheme ${ }^{[73]}$ wherein the $g$ tensors of the radicals are assumed to be isotropic, or the anisotropy averages out on the timescale of the geminate recombination. Further development of the theory would require that the spin-orbit mixed nature of the radical-pair substates and the anisotropy of the spin Hamiltonian together with the dynamics of molecular rotation are explicitly taken into account. ${ }^{[70]}$

\section{Experimental Section}

Reagents and Sample Solutions: Standard phosphate buffer solutions of $\mathrm{pH} 7.6$ were prepared from deionized water, $\mathrm{K}_{2} \mathrm{HPO}_{4}$, and $\mathrm{KH}_{2} \mathrm{PO}_{4}\left(\geq 99.0 \%\right.$, Fluka) by mixing $50 \mathrm{~mm}$ solutions of $\mathrm{K}_{2} \mathrm{HPO}_{4}$ and $\mathrm{KH}_{2} \mathrm{PO}_{4}$ in a ratio of 9:1 (v:v) and adjusting to $\mathrm{pH} 7.6$ by controlled addition of the deficient component using a glass electrode. To eliminate traces of transition metal ions, diethylenetriamine pentaacetic acid (DTPA, $\geq 99.0 \%$, Fluka) was added at a concentration of $1 \mathrm{~mm}$. The buffer solution was stored at $-20^{\circ} \mathrm{C}$. Stock $1 \mathrm{~mm}$ solutions of SIN-1 (3-morpholinosydnonimine hydrochloride, Sigma) in pH 7.6 buffer and dihydrorhodamine 123 (DHR, Sigma, $\geq 95 \%$ ) in acetonitrile were also stored at $-20^{\circ} \mathrm{C}$ after preparation.

Determination of $\mathrm{ONOO}^{-}$: The yield of $\mathrm{ONOO}^{-}$on decomposition of SIN-1 was determined by means of the reaction of $\mathrm{ONOO}^{-}$with DHR producing rhodamine $123(\mathrm{RH})$ with a characteristic optical absorption band at $500 \mathrm{~nm}\left(\varepsilon=78500 \mathrm{M}^{-1} \mathrm{~cm}^{-1}\right)$ as a stable product. To determine the amount of accumulated RH, optical absorption spectra of the incubation mixtures were taken, and the absorbance in the maximum of the RH absorption band converted to $\mathrm{RH}$ concentration. For measuring the yield of RH in a set of samples from one magnetic-field experiment, the absorption of each sample was measured in the same cuvette (path length $1 \mathrm{~mm}$ ).
After each absorption measurement, the cuvette was rinsed with water, then with acetone (chromatography grade), and finally blown dry with a gentle flow of dry air or nitrogen. The spectra were taken on a Shimadzu UV-2401 or a Varian CARY 50 UVNis spectrometer.

Magnetic-Field Experiments: For measurements at 4.7 T (Novosibirsk), a $200 \mathrm{MHz}$ NMR magnet (Bruker Avance 200) was used, and for measurements at zero field and between 7 and $18 \mathrm{~T}$ (Konstanz) a $\mathrm{Nb}_{3} \mathrm{Sn}$ superconducting magnet (Oxford Instruments) was used. In the latter magnet, the field could be continuously varied from 0 to $18 \mathrm{~T}$. For each magnetic-field experiment, $5.0 \mathrm{~mL}$ of a fresh incubation mixture was prepared after allowing the stock solutions taken from the refrigerator to thermalize. For example, for a reaction solution to be $50 \mu \mathrm{M}$ in SIN-1 and DHR, $4685 \mu \mathrm{L}$ of buffer were mixed with $250 \mu \mathrm{L}$ of SIN-1 and $65 \mu \mathrm{L}$ of DHR stock solutions. From this mixture, $400 \mu \mathrm{L}$ each was transferred into 10-12 $1600 \mu \mathrm{L}$ Eppendorf cups, of which 5-6 samples were incubated in the field and 5-6 samples were left as controls. In each experiment, the exposed group of samples was placed in the bore of the magnet in the region of maximum field, and the group of control samples was placed at the top opening of the bore, where the measured magnetic field was about $1 \mathrm{mT}$ for the $4.75 \mathrm{~T}$ field in the Bruker magnet. Such a low field can be neglected for the purposes of this study, but the described placement of control samples helped reduce the difference in the temperatures of the exposed and control samples down to several tenths of a Kelvin. For the experiments in the variable-field magnet, a special sample holder for the two groups of samples was designed. For both control and in-field samples a tandem sample holder consisting of two cylindrical parts, each with openings to hold three Eppendorf cups was available. All cylinders were threaded on a system of three brass rods. The distance between the in-field tandem and the control tandem was about $1 \mathrm{~m}$. When the in-field tandem reached the center of the field the control was just above the top of the opening of the magnet bore. The fringe field at the control amounted to about $0.3 \%$ of the central field, which causes a negligible error for the MFE. The cylindrical sample holders had a water jacket and were connected to a thermostat. (for details, see the Supporting Information). In each of the two tandem compartments of the holder one position was occupied by a dummy sample with a thermocouple junction to control the temperature. The temperature difference between control and in-field sample holders was recorded throughout the reaction period, and it was made sure that the temperature difference between them was kept within $0.3^{\circ} \mathrm{C}$. The thermostat also allowed experiments to be performed at elevated temperature $\left(40^{\circ} \mathrm{C}\right)$. Since $\mathrm{SIN}-1$ decomposition and DHR-123 oxidation are light-sensitive ${ }^{[45,81]}$ the samples were kept in the dark by wrapping the Eppendorf cups in aluminum foil. After preparing the reaction mixtures, the samples were immediately transferred to the magnet and were allowed to react for $24 \mathrm{~h}$ before analyzing the yield of $\mathrm{RH}$.

Evaluation of the Experimental Data: The magnetic-field effect was calculated as the relative difference between the average absorbances of RH in the two groups of samples. To exclude doubtful results, the $Q$ criterion with a confidence probability of $P=0.95$ was applied, and at most one, if any, outlier value was excluded from each sampling. The standard deviation of the determined magnetic-field effect was calculated by using the law of error propagation. The magnetic-field effect in a single experiment and its standard deviation were calculated by using Equations (37) and (38): 
$\varphi=\frac{\bar{A}_{\mathrm{m}}-\bar{A}_{\mathrm{c}}}{\bar{A}_{\mathrm{c}}}$

$s_{\varphi}=\sqrt{\left(\frac{s_{m}}{\bar{A}_{m}}\right)^{2}+\left(\frac{s_{c}}{\bar{A}_{c}}\right)^{2}} \times(\varphi+1)$

where $\varphi$ is the sought magnetic-field effect, $s_{\varphi}$ its standard deviation, $\bar{A}_{\mathrm{m}}$ and $\bar{A}_{\mathrm{c}}$ are the average optical densities of the groups of samples in the maximum of the $\mathrm{RH}-123$ absorption band $(500 \mathrm{~nm}$ ) for exposed and control samples, respectively, and $s_{m}$ and $s_{c}$ are their respective standard deviations. The average magnetic-field effect and its standard deviation over several experiments at the same magnetic field were calculated by using Equations (39) and (40):

$\bar{\varphi}\left(B_{0}\right)=\frac{\sum \bar{\varphi}_{i}\left(B_{0}\right)}{m}$

$S_{\bar{\varphi}}\left(B_{0}\right)=\sqrt{\frac{\sum\left[\bar{\varphi}_{i}\left(B_{0}\right)-\bar{\varphi}\left(B_{0}\right)\right]^{2}}{m-1}}$

where the $\bar{\varphi}_{i}$ are the average MFEs of the individual sets of altogether $m$ experiments at the same field.

\section{Acknowledgements}

This work was supported by the University of Konstanz, Deutsche Forschungsgemeinschaft, International Research Training Group 667, the Council for Grants of the President of the Russian Federation for Support of leading scientific Schools (grant no. NSh 4249.2010.3), and the Division of Chemistry and Materials Science, RAS (grant no. 5.1.6, 2010). We thank Prof. Dr. G. Maret for extensive access to the high-field superconducting magnet, Nathan Isert for his assistance with the magnetic-field experiments and Prof. Dr. N. Lukzen and Prof. Dr. W. Koppenol for helpful discussions.

Keywords: magnetic properties - nitrogen oxides • radical reactions · reaction mechanisms · spin chemistry

[1] S. Johnsen, K. J. Lohmann, Phys. Today 2008, 61, 29-35.

[2] S. Johnsen, K. J. Lohmann, Nat. Rev. Neurosci. 2005, 6, 703-712.

[3] W. Wiltschko, R. Wiltschko, J. Comp. Physiol. A 2005, 191, 675-693.

[4] J. L. Kirschvink, M. M. Walker, C. E. Diebel, Curr. Opin. Neurobiol. 2001, 11, 462-467.

[5] Biophysical Effects of Steady Magnetic Fields, Vol. 11 (Eds.: G. Maret, J. Kiepenheuer, N. Boccara), Springer, Berlin, 1986.

[6] K. M. Salikhov, Y. N. Molin, R. Z. Sagdeev, A. L. Buchachenko, Spin Polarization and Magnetic Effects in Radical Reactions, Elsevier, Amsterdam, 1984.

[7] U. E. Steiner, T. Ulrich, Chem. Rev. 1989, 89, 51-147.

[8] Dynamic Spin Chemistry. Magnetic Controls and Spin Dynamics of Chemical Reactions, (Eds.: S. Nagakura, H. Hayashi, T. Azumi), Kodansha and Wiley, Tokyo and New York, 1998.

[9] H. Hayashi, Introduction to Dynamic Spin Chemistry: Magnetic Field Effects on Chemical and Biochemical Reactions, World Scientific Publishing, Singapore, 2004.

[10] J. R. Woodward, T. J. Foster, A. R. Jones, A. T. Salaoru, N. S. Scrutton, Biochem. Soc. Trans. 2009, 37, 358-362.

[11] S. G. Boxer, C. E. D. Chidsey, M. G. Roelofs, Annu. Rev. Phys. Chem. 1983, 34, 389-417.

[12] A. Hoff, Photochem. Photobiol. 1986, 43, 727-745.
[13] A. R. Jones, S. Hay, J. R. Woodward, N. S. Scrutton, J. Am. Chem. Soc. $2007,129,15718-15727$

[14] M. S. Afanasyeva, M. B. Taraban, P. A. Purtov, T. V. Leshina, C. B. Grissom, J. Am. Chem. Soc. 2006, 128, $8651-8658$

[15] A. L. Buchachenko, Pure Appl. Chem. 2000, 72, 2243-2258.

[16] D. R. Blake, R. E. Allen, J. Lunec, Br. Med. Bull. 1987, 43, 371 - 385.

[17] S. Moncada, R. M. J. Palmer, E. A. Higgs, Pharm. Rev. 1991, 43, 109-142.

[18] M. A. Marletta, M. A. Tayeh, J. M. Hevel, BioFactors 1990, 2, 219-225.

[19] The commonly used name peroxinitrite is not recommended by IUPAC.

[20] R. Radi, J. S. Beckmann, K. M. Bush, B. A. Freeman, J. Biol. Chem. 1991, 266, 4244-4250.

[21] M. Trujillo, M. Naviliat, M. N. Alvarez, G. Peluffo, R. Radi, Analusis 2000 28, 518-527.

[22] G. Ferrer-Sueta, R. Radi, ACS Chem. Biol. 2009, 4, 161-177.

[23] R. J. Miller, D. Feller, J. Phys. Chem. 1994, 98, 10375.

[24] An external magnetic field can also reduce the rate of spin relaxation but this is not the case if the correlation time of the stochastic process driving the relaxation is shorter than the Larmor period. The latter applies for spin-rotational relaxation, as should be the case for NO and $\mathrm{O}_{2}{ }^{*-}$.

[25] P. Gilch, F. Pöllinger-Dammer, C. Musewald, M. E. Michel-Beyerle, U. E. Steiner, Science 1998, 281, 982-984.

[26] I. A. Solov'yov, K. Schulten, Biophys. J. 2009, 96, 4804-4813.

[27] H. J. Hogben, O. Efimova, N. Wagner-Rundell, C. R. Timmel, P. J. Hore, Chem. Phys. Lett. 2009, 480, 118-122.

[28] B. Bhattacharjee, R. Das, Mol. Phys. 2007, 105, 1053-1057.

[29] M. D. E. Forbes, N. V. Lebedeva, C. R. White, T. K. Chen, R. Macarthur, P. Caregnato, T. E. Hill, Mol. Phys. 2007, 105, 2127-2136.

[30] T. Y. Karogodinà, S. V. Sergeeva, D. V. Stass, Appl. Magn. Reson. 2009, 36, 195-208.

[31] Y. Asahi, K. Shinozaki, M. Nagaoka, Chem. Pharm. Bull. 1971, 19, 10791088.

[32] M. Kirsch, H. de Groot, J. Biol. Chem. 1999, 274, 24664-24670.

[33] M. M. Tarpey, D. A. Wink, M. B. Grisham, Am. J. Physiol. Regul. Integr. Comp. Physiol. 2004, 286, R431-R444.

[34] M. Feelisch, E. Noack, Eur. J. Pharmacol. 1987, 139, 19-30.

[35] H. Bohn, K. Schönafinger, J. Cardiovasc. Pharmacol. 1989, 14, S6-S12.

[36] M. Feelisch, J. Ostrowski, E. Noack, J. Cardiovasc. Pharmacol. 1989, 14, $\mathrm{S} 13-\mathrm{S} 22$.

[37] R. J. Singh, N. Hogg, J. Joseph, E. Konorev, B. Kalyanaraman, Arch. Biochem. Biophys. 1999, 361, 331-339.

[38] T. Nauser, W. H. Koppenol, J. Phys. Chem. A 2002, 106, 4084-4086.

[39] In a highly polar solvent such as water, the Onsager length (the distance at which the Coulomb interaction between two charged particles equals $-k_{B} T$ of about $7 \AA$ is small enough that its effect on the diffusive separation can be considered as negligible for our purpose, the more so since radical pairs created by electron transfer can originate with separations larger than defined by contact.

[40] H. Botti, M. N. Moller, D. Steinmann, T. Nauser, W. H. Koppenol, A. Denicola, R. Radi, J. Phys. Chem. B 2010, 114, 16584-16593.

[41] J. M. Deutch, J. Chem. Phys. 1972, 56, 6076-6081.

[42] A. Gomes, E. Fernandes, J. L. F. C. Lima, J. Fluoresc. 2006, 16, 119-139.

[43] N. W. Kooy, J. A. Royall, J. S. Beckmann, Free Radical Biol. Med. 1994, 16, $149-156$.

[44] J. A. Royall, H. Ischiropoulos, Arch. Biochem. Biophys. 1993, 302, 348 355.

[45] L. M. Henderson, J. B. Chappell, Eur. J. Biochem. 1993, 217, 973-980.

[46] S. S. Kumar, K. I. Priyadarsini, K. B. Sainis, J. Agric. Food Chem. 2004, 52, $139-145$.

[47] J. Glebska, W. H. Koppenol, Free Radical Biol. Med. 2003, 35, 676-682.

[48] D. Jourd'heuil, F. L. Jourd'heuil, P. S. Kutchukian, R. A. Musah, D. A. Wink, M. B. Grisham, J. Biol. Chem. 2001, 276, 28799-28805.

[49] If the $g$ tensors are anisotropic, the magnetic-field-induced mixing can also occur between the triplet components $T_{+}, T_{-}$and the singlet state $\mathrm{S}$ of the radical pair.

[50] Y. Tanimoto, H. Hayashi, S. Nagakura, Chem. Phys. Lett. 1976, 41, 267269.

[51] W. Känzig, M. H. Cohen, Phys. Rev. Lett. 1959, 3, 509-510.

[52] G. Herzberg, Molecular Spectra and Molecular Structure, Vol. I., 2nd ed., Van Nostrand Reinhold, New York, 1950.

[53] M. C. R. Symons, Magn. Reson. Chem. 1995, 33, S3-S13. 
[54] C. L. Gardner, M. A. Weinberger, Can. J. Chem. 1970, 48, 1318-1322.

[55] T. Rudolf, A. Pöppl, W. Hofbauer, D. Michel, Phys. Chem. Chem. Phys. 2001, 3, 2167-2173.

[56] H. R. Zeller, W. Känzig, Helv. Phys. Acta 1967, 40, 845-855.

[57] H. J. Bielski, J. M. Gebicki, J. Am. Chem. Soc. 1982, 104, 796-798.

[58] P. A. Narayana, D. Suryanarayana, L. Kevan, J. Am. Chem. Soc. 1982, 104, 3552-3555.

[59] M. J. Weber, J. A. Kelley, S. B. Nielsen, P. Ayotte, J. M. A. , Science 2000, 287, 2461-2463.

[60] P. W. Atkins, D. Kivelson, J. Chem. Phys. 1966, 44, 169-174.

[61] D. Kivelson, in Rotational Dynamics of Small and Macromolecules (Eds.: T. Dorfmüller, R. Pecora), Springer-Verlag, Heidelberg, 1987.

[62] C.-M. Hu, R. Zwanzig, J. Chem. Phys. 1974, 60, 4354-4357.

[63] U. E. Steiner, Y. A. Serebrennikov, J. Chem. Phys. 1994, 100, 7503-7507.

[64] Y. A. Serebrennikov, U. E. Steiner, J. Chem. Phys. 1994, 100, 7508-7514.

[65] A. Carrington, A. D. McLachlan, Introduction to Magnetic Resonance, Chapman and Hall, London, 1979.

[66] H. W. Spiess, D. Schweitzer, U. Haeberlen, K. H. Hausser, J. Magn. Reson. 1971, 5, $101-108$.

[67] H. J. Bakker, Y. L. A. Rezus, R. L. A. Timmer, J. Phys. Chem. A 2008, 112, $11523-11534$.

[68] B. Brocklehurst, J. Chem. Soc. Faraday Trans. 2 1979, 75, 123-140.

[69] H. M. McConnell, J. Chem. Phys. 1961, 34, 13-16.

[70] We note here that with $g$ tensors deviating strongly from the isotropic $g_{e}$ the spin situation is in fact more complicated because 1) the $g$ tensor refers to an effective spin by formally treating spin-orbit-coupled Kramers doublet like normal spin-only doublets. In radical pairs comprising radicals with strongly anisotropic $g$ tensor, spin states with pure $S$ or $T$ character are not separable. 2 ) In such radical pairs the Zeeman Hamiltonian not only mixes the (formal) $\mathrm{S}$ and $\mathrm{T}_{0}$ states but also $S$ with (the formal) $T_{+}$and $T_{-}$states (cf. U. E. Steiner, D. Bürßner, $Z$. Phys. Chem. N. F. 1990, 169, 159-180). These aspects would have to be properly taken into account in a more in-depth treatment. In the pres- ent work, however, we confine ourselves to a semiquantitative treatment by adopting the simplifying assumption of pure spin states and rapid averaging of the $g$-tensor anisotropy by rotational motion of the radicals.

[71] K. A. McLauchlan, U. E. Steiner, Mol. Phys. 1991, 73, 241-263.

[72] R. G. Mints, A. A. Pukhov, Chem. Phys. 1984, 87, 467-472.

[73] E. V. Gorelik, N. N. Lukzen, R. Z. Sagdeev, U. E. Steiner, Chem. Phys. 2000, 262, 303.

[74] A. I. Burshtein, N. N. Lukzen, J. Chem. Phys. 1995, 103, 9631-9641.

[75] In the reasoning presented here, strict spin conservation in the formation of the singlet recombination product is assumed. Note, though, that spin-orbit coupling might relax this spin-selection rule to some extent. It will be of interest to investigate in more detail what the contribution of such a "spin-forbidden" recombination might be in the formation of $\mathrm{ONOO}^{-}$from $\mathrm{NO}$ and $\mathrm{O}_{2}^{--}$.

[76] The width $\delta$ of the reaction zone determines to what extent relaxation takes place within that zone in addition to the relaxation taking place in the "diffusional zone" from which geminate re-encounters can occur. If $\tau_{\mathrm{s}}>3 \tau_{\mathrm{c}}$ and $\delta<0.3 \AA$, more than $80 \%$ of relaxation occurs in the diffusional zone

[77] K. Schulten, H. Staerk, A. Weller, H. J. Werner, B. Nickel, Z. Phys. Chem. $1976,101,371-390$

[78] B. H. J. Bielski, D. E. Cabelli, R. L. Arudi, A. B. Ross, J. Phys. Chem. Ref. Data 1985, 14, $1041-1100$.

[79] V. G. Kharitonov, A. R. Sundquist, V. S. Sharma, J. Biol. Chem. 1994, 269, $5881-5883$

[80] J. Butler, B. Halliwell, Arch. Biochem. Biophys. 1982, 218, 174-178.

[81] T. Ullrich, S. Oberle, A. Abate, H. Schröder, FEBS Lett. 1997, 406, 66.68. 